\title{
GENERIC MODAL CUT ELIMINATION APPLIED TO CONDITIONAL LOGICS
}

\author{
DIRK PATTINSON ${ }^{a}$ AND LUTZ SCHRÖDER ${ }^{b}$ \\ ${ }^{a}$ Department of Computing, Imperial College London \\ ${ }^{b}$ DFKI Bremen and Department of Computer Science, Universität Bremen
}

\begin{abstract}
We develop a general criterion for cut elimination in sequent calculi for propositional modal logics, which rests on absorption of cut, contraction, weakening and inversion by the purely modal part of the rule system. Our criterion applies also to a wide variety of logics outside the realm of normal modal logic. We give extensive example instantiations of our framework to various conditional logics. For these, we obtain fully internalised calculi which are substantially simpler than those known in the literature, along with leaner proofs of cut elimination and complexity. In one case, conditional logic with modus ponens and conditional excluded middle, cut elimination and complexity were explicitly stated as open in the literature.
\end{abstract}

\section{INTRODUCTION}

Cut elimination, originally conceived by Gentzen [7, is one of the core concepts of proof theory and plays a major role in particular for algorithmic aspects of logic, including the complexity of automated reasoning and, via interpolation, modularity issues. The large number of logical calculi that are currently in use, in particular in various areas of computer science, motivates efforts to define families of sequent calculi that cover a variety of logics and admit uniform proofs of cut elimination, enabled by suitable sufficient conditions. Here, we present such a method for modal sequent calculi that applies to possibly non-normal normal modal logics, which appear, e.g. in concurrency and knowledge representation. We use a separation of the modal calculi into a fixed underlying propositional part and a modal part. The core of our criterion, that we call the absorption of cut, stipulates that an application of the cut rule to conclusions of modal rules can be replaced by a single rule application. This concept generalises the notion of resolution closed rule set [14, 18], dropping the assumption that the logic at hand is rank-1, i.e. axiomatised by formulas in which the nesting depth of modal operators is uniformly equal to 1 (such as $K$ ).

1998 ACM Subject Classification: F.4.1, I.2.3.

Key words and phrases: Modal Logic, Proof Theory, Cut Elimination, Conditional Logic.

${ }^{a}$ Partially supported by EPSRC grant EP/F031173/1.

${ }^{b}$ Work performed as part of the DFG project SCHR 1118/5-1. 
Our method is reasonably simple and intuitive, and nevertheless applies to a wide range of modal logics. While we use normal modal logics such as $K$ and $T$ as running examples to illustrate our concepts at the time of introduction, our main applications are conditional logics, which have a binary modal operator $\Rightarrow$ read as a non-monotonic implication (unlike default logics, conditional logics allow nested non-monotonic implications). In particular, we prove cut-elimination (hence, since the generic systems under consideration are analytic, the subformula property) for the conditional logic CK and all its extensions by any of the axioms conditional modus ponens (MP) $(A \Rightarrow B) \rightarrow A \rightarrow B$, where $\rightarrow$ denotes material implication, conditional excluded middle (CEM) $(A \Rightarrow B) \vee(A \Rightarrow \neg B)$, and conditional identity (ID) $A \Rightarrow$ $A$ using our generic procedure. An easy analysis of proof search in the arising cut-free calculi moreover establishes that the satisfiability problem of each of these logics is in PSPACE. This is a tight bound for logics not containing CEM, whereas the provability problem in CKCEM and CKIDCEM can be solved in coNP, as we show by a slightly adapted algorithmic treatment of our calculus using a dynamic programming approach in the spirit of [20]. We point out that while (different) cut-free labelled sequent calculi for CK, CKMP, CKCEM, and some further conditional logics, as well as the ensuing upper complexity bounds, have previously been presented by Olivetti et al., the corresponding issues for CKMPCEM have explicitly been left as open problems [13]; moreover, our coNP upper bounds for CKCEM and CKIDCEM improve previous upper PSPACE bounds.

Related work. A set of sufficient conditions for a sequent calculus to admit cut elimination and a subsequent analysis of the complexity of cut elimination (not of proof search) is presented in [16. The range of application of this method is very wide and encompasses, e.g. first-order logic, the modal logic $S 4$, linear logic, and intuitionistic propositional logic. This generality is reflected in the fact that the method as a whole is substantially more involved than ours. A simpler method for a different and comparatively restrictive class of calculi, so-called canonical calculi, is considered in [1]; this method does not apply to typical modal systems, as it considers only so-called canonical rules, i.e., left and right introduction rules for connectives which permit adding a common context simultaneously in the premise and the conclusion. (In fact, it might be regarded as the essence of modal logic that its rules fail to be canonical, e.g. the necessitation rule $A / \square A$ does not generalise to $\Gamma, A / \Gamma, \square A$ for a sequent $\Gamma$.) Moreover, the format of the rules in op.cit. does not allow for the introduction of more than one occurrence of a logical connective, which is necessary even for the most basic modal logics. The same applies to [5]. In [4], logical rules are treated on an individual basis, which precludes the treatment of cuts between two rule conclusions. Overall, our notion of absorption is substantially more general when compared to similar notions in the papers discussed above, which stipulate that cuts between left and right rules for the same connective are absorbed by structural rules. In our own earlier work [14], we have considered a special case of the method presented here in the restricted context of rank-1 logics; in particular, these results did not cover logics such as $K 4$, CKMP, or CKMPCEM.

This work is an extended and revised version of [15].

\section{Preliminaries and Notation}

A modal similarity type (or modal signature) is a set $\Lambda$ of modal operators with associated arities that we keep fixed throughout the paper. Given a set $V$ of propositional 

(N) $\frac{A}{\square A}$
(D) $\square(A \rightarrow B) \rightarrow(\square A \rightarrow \square B)$
(4) $\square A \rightarrow$
$(\mathrm{R}) \square A \rightarrow A$

Figure 1: Axioms and Rules of modal Hilbert Systems

variables, the set $\mathcal{F}(\Lambda)$ of $\Lambda$-formulas is given by the grammar

$$
\mathcal{F}(\Lambda) \ni A, B::=\perp|p| \neg A|A \wedge B| \varnothing\left(A_{1}, \ldots, A_{n}\right)
$$

where $p \in V$ and $\varnothing \in \Lambda$ is $n$-ary. We use standard abbreviations of the other propositional connectives $T, \vee$ and $\rightarrow$. A $\Lambda$-sequent is a finite multiset of $\Lambda$-formulas, and the set of $\Lambda$ sequents is denoted by $\mathrm{S}(\Lambda)$. We write the multiset union of $\Gamma$ and $\Delta$ as $\Gamma, \Delta$ and identify a formula $A \in \mathcal{F}(\Lambda)$ with the singleton sequent containing only $A$. If $S \subseteq \mathcal{F}(\Lambda)$ is a set of formulas, then an $S$-substitution is a mapping $\sigma: V \rightarrow S$. We denote the result of uniformly substituting $\sigma(p)$ for $p$ in a formula $A$ by $A \sigma$. This extends pointwise to $\Lambda$-sequents so that $\Gamma \sigma=A_{1} \sigma, \ldots, A_{n} \sigma$ if $\Gamma=A_{1}, \ldots, A_{n}$. If $S \subseteq \mathcal{F}(\Lambda)$ is a set of $\Lambda$-formulas and $A \in \mathcal{F}(\Lambda)$, we say that $A$ is a propositional consequence of $S$ if there exist $A_{1}, \ldots, A_{n} \in S$ such that $A_{1} \wedge \cdots \wedge A_{n} \rightarrow A$ is a substitution instance of a propositional tautology. We write $S \vdash_{\mathrm{PL}} A$ if $A$ is a propositional consequence of $S$ and $A \vdash_{\mathrm{PL}} B$ for $\{A\} \vdash_{\mathrm{PL}} B$ for the case of single formulas.

\section{Modal Deduction Systems}

To facilitate the task of comparing the notion of provability in both Hilbert and Gentzen type proof systems, we introduce the following notion of a proof rule that can be used, without any modifications, in both systems.

Definition 3.1. A $\Lambda$-rule is of the form $\frac{\Gamma_{1} \ldots \Gamma_{n}}{\Gamma_{0}}$ where $n \geq 0$ and $\Gamma_{0}, \ldots, \Gamma_{n}$ are $\Lambda$-sequents. The sequents $\Gamma_{1}, \ldots, \Gamma_{n}$ are the premises of the rule and $\Gamma_{0}$ its conclusion. A rule $\overline{\Gamma_{0}}$ without premises is called a $\Lambda$-axiom, which we denote by just its conclusion, $\Gamma_{0}$. A rule set is just a set of $\Lambda$-rules, and we say that a rule set R is substitution closed, if $\Gamma_{1} \sigma \ldots \Gamma_{n} \sigma / \Gamma_{0} \sigma \in \mathrm{R}$ whenever $\Gamma_{1} \ldots \Gamma_{n} / \Gamma_{0} \in \mathrm{R}$ and $\sigma: V \rightarrow \mathcal{F}(\Lambda)$ is a substitution.

In view of the sequent calculi that we introduce later, we read sequents disjunctively. Consequently, a rule $\Gamma_{1} \ldots, \Gamma_{n} / \Gamma_{0}$ can be used to prove the disjunction of $\Gamma_{0}$, provided that $\bigvee \Gamma_{i}$ is provable, for all $1 \leq i \leq n$. We emphasise that a rule is an expression of the object language, i.e. it does not contain meta-linguistic variables. As such, it represents a specific deduction step rather than a family of possible deductions, which helps to economise on syntactic categories. In our examples, concrete rule sets are presented as instances of rule schemas.

Example 3.2. For the modal logics $K, K 4$ and $T$, we fix the modal signature $\Lambda=\{\square\}$ consisting of a single modal operator $\square$ with arity one. The language of conditional logic is given by the similarity type $\Lambda=\{\Rightarrow\}$ where the conditional arrow $\Rightarrow$ has arity 2 . We use infix notation and write $A \Rightarrow B$ instead of $\Rightarrow(A, B)$ for $A, B \in \mathcal{F}(\Lambda)$. Formulas $A \Rightarrow B$ are interpreted as various forms of conditionals, e.g. default implication 'if $A$ then normally $B$ ', relevant implication and others, depending on the choice of semantics and imposed logical principles. Deduction over modal and conditional logics are governed by the following rule sets: 


$$
\begin{gathered}
(\mathrm{RCEA}) \frac{A \leftrightarrow A^{\prime}}{(A \Rightarrow B) \leftrightarrow\left(A^{\prime} \Rightarrow B\right)} \quad(\mathrm{RCK}) \frac{B_{1} \wedge \cdots \wedge B_{n} \rightarrow B}{\left(A \Rightarrow B_{1}\right) \wedge \cdots \wedge\left(A \Rightarrow B_{n}\right) \rightarrow(A \Rightarrow B)} \\
(\mathrm{ID}) A \Rightarrow A \quad(\mathrm{MP})(A \Rightarrow B) \rightarrow(A \rightarrow B) \quad(\mathrm{CEM})(A \Rightarrow B) \vee(A \Rightarrow \neg B)
\end{gathered}
$$

Figure 2: Axioms and Rules of conditional Hilbert Systems

(1) The rule set $\mathrm{K}$ associated to the modal logic $K$ consists of all instances of the necessitation rule $(\mathrm{N})$ and the distribution axiom (D) in Figure 1. The rule sets that axiomatise the logics $T$ and $K 4$ arise by extending this set with the reflexivity axiom (R) and the (4)-axiom, respectively. We reserve the name $(T)$ for the reflexivity rule in a cut-free system.

(2) The basic conditional logic is the system CK of [3], axiomatised by the rule set that consists of all instances of (RCEA) and (RCK) in Figure 2, The system CK constitutes a minimal set of properties to be reasonably expected of any conditional, however nonstandard: replacement of equivalents in the left hand argument, and compatibility with conjunction in the right-hand argument. Additional properties are typically imposed when more specific interpretations of $\Rightarrow$ are intended. E.g. the basic properties of $\Rightarrow$ viewed as a default implication are given by Burgess' System $\mathcal{S}$ [2], which is related to the well-known KLM postulates of default reasoning [11]. A treatment of System $\mathcal{S}$ using methods of the present work and [14] is presented in [19]. Here, we consider several other standard axioms, namely identity (ID), conditional modus ponens (MP) and conditional excluded middle (CEM), also given in Figure 2, We denote the corresponding extensions of CK by juxtaposition of the respective axioms, e.g. CKMPCEM contains the rules for $\mathrm{CK}$ and the axioms (MP) and (CEM). As indicated above, whether or not these axioms are accepted depends on the intended reading of the conditional. E.g., modus ponens is a reasonable principle for interpretations of the conditional as a relevant implication or as a counterfactual, but not for default implication; conditional excluded middle is a controversially discussed property of the subjunctive conditional [6]. The identity axiom, while accepted for many interpretations of the conditional including as default implication, is typically rejected for causal interpretations [8].

Rules with more than one premise arise through saturation of a given rule set under cut that, e.g. leads to the rules $\left(\mathrm{CK}_{g}\right)$ and $\left(\mathrm{MP}_{g}\right)$ presented in Section 6 ,

In order to make the mapping between Hilbert-style and Gentzen-style systems easier, we take the derivability predicate of a Hilbert-system to be induced by a set of $\Lambda$-rules and read each sequent as the disjunction of its elements. The notion of deduction in modal Hilbert systems then takes the following form.

Definition 3.3. Suppose R is a set of rules. The set of R-derivable formulas in the Hilbertsystem given by $\mathrm{R}$ is the least set of formulas that

- contains $A \sigma$ whenever $A$ is a propositional tautology and $\sigma$ is a substitution

- contains $B$ whenever it contains $A$ and $A \rightarrow B$

- contains $\bigvee \Gamma_{0}$ whenever it contains $\bigvee \Gamma_{1}, \ldots, \bigvee \Gamma_{n}$ and $\frac{\Gamma_{1} \ldots \Gamma_{n}}{\Gamma_{0}} \in \mathrm{R}$.

We write $\mathrm{HR} \vdash A$ if $A$ is R-derivable. 
In other words, the set of derivable formulas is the least set that contains propositional tautologies, is closed under uniform substitution, modus ponens and application of rules. We will later consider Hilbert systems that induce the same provability predicate based on the following notion of admissibility.

Definition 3.4. A rule set $\mathrm{R}^{\prime}$ is admissible in $\mathrm{HR}$ if $\mathrm{HR} \vdash A \Longleftrightarrow \mathrm{H}\left(\mathrm{R} \cup \mathrm{R}^{\prime}\right) \vdash A$ for all formulas $A \in \mathcal{F}(\Lambda)$. Two rule sets $\mathrm{R}, \mathrm{R}^{\prime}$ are equivalent if $\mathrm{R}$ is admissible in $\mathrm{HR}^{\prime}$ and $\mathrm{R}^{\prime}$ is admissible in $\mathrm{HR}$.

In words, $R^{\prime}$ is admissible in $H R$ if adding the rules $R^{\prime}$ to those of $R$ leaves the set of provable formulas unchanged. We note the following trivial, but useful consequence of admissibility.

Lemma 3.5. Let $\mathrm{R}$ and $\mathrm{R}^{\prime}$ be equivalent, and let $A \in \mathcal{F}(\Lambda)$. Then $\mathrm{HR} \vdash A$ iff $\mathrm{HR}^{\prime} \vdash A$.

The next proposition establishes a rudimentary form of proof normalisation in Hilbert systems and is the key for proving equivalence of Hilbert and Gentzen-type systems. We show that every derivable formula in a Hilbert-sytem is a propositional consequence of conclusions of rules with provable premises, which stratifies proofs into rule application and propositional reasoning and avoids modus ponens.

Proposition 3.6. Suppose that $S$ is the least set of formulas that is closed under propositional consequences of rule conclusions, that is, $S$ contains $A \in \mathcal{F}(\Lambda)$ whenever there are rules $\Theta_{1} / \Gamma_{1}, \ldots, \Theta_{n} / \Gamma_{n} \in \mathrm{R}$ and substitutions $\sigma_{1}, \ldots, \sigma_{n}: V \rightarrow \mathcal{F}(\Lambda)$ such that $\bigvee \Delta \sigma_{i} \in S$ for all $\Delta \in \Theta_{i}(i=1, \ldots, n)$, and $\left\{\bigvee \Gamma_{1} \sigma, \ldots, \bigvee \Gamma_{n} \sigma\right\} \vdash_{\mathrm{PL}} A$.

Then $S$ coincides with the set of derivable formulas in the Hilbert-calculus induced by $\mathrm{R}$, that is $S=\{A \in \mathcal{F}(\Lambda) \mid \mathrm{HR} \vdash A\}$.

Proof. We write $\mathrm{HT}(\mathrm{R})=\{A \in \mathcal{F}(\Lambda) \mid \mathrm{HR} \vdash A\}$ for the set of provable formulas in HR. The inclusion $S \subseteq \mathrm{HT}(\mathrm{R})$ is immediate as $\mathrm{HT}(\mathrm{R})$ contains propositional tautologies, is closed under uniform substitution and modus ponens. For the reverse inclusion we show that $S$ is closed under R-derivability as considered in Definition 3.3 .

This is clear for all cases (propositional tautologies, uniform substitutions, rule application) except possibly modus ponens. So assume that $\mathrm{HR} \vdash A \rightarrow B$ and $\mathrm{HR} \vdash A$. By induction hypothesis, there are

- Rules $\Theta_{1} / \Gamma_{1}, \ldots, \Theta_{n} / \Gamma_{n}$ and substitutions $\sigma_{1}, \ldots, \sigma_{n}$ such that $\left\{\bigvee \Gamma_{1} \sigma_{1}, \ldots, \bigvee \Gamma_{n} \sigma_{n}\right\} \vdash_{\mathrm{PL}}$ $A \rightarrow B$

- Rules $\Sigma_{1} / \Delta_{1}, \ldots, \Sigma_{k} / \Delta_{k}$ and substitutions $\tau_{1}, \ldots, \tau_{k}$ such that $\left\{\bigvee \Delta_{1} \tau_{1}, \ldots, \bigvee \Delta_{k} \tau_{k}\right\} \vdash_{\mathrm{PL}}$ $A$

and moreover $\bigvee \Xi \sigma \in S$ whenever $\Xi \in \Theta_{1}, \ldots, \Theta_{n}, \Sigma_{1}, \ldots, \Sigma_{k}$. The claim follows, as $\left\{\Gamma_{1} \sigma_{1}, \ldots, \Gamma_{n} \sigma_{n}, \Delta_{1} \tau_{1}, \ldots, \Delta_{k} \tau_{k}\right\} \vdash_{\mathrm{PL}} B$.

In other words, in a modal Hilbert system, each provable formula is a propositional consequence of rule conclusions with provable premises. This result forms the basis of our comparison of Hilbert and Gentzen systems. The point to note is that in a Hilbert system, provable formulas are propositional consequences of zero or more rule conclusions with provable premises. The propositional reasoning that is applied when showing that the set of conclusions implies a formula generally uses the cut-rule. As a consequence, the need for cut vanishes if there is no need to apply propositional reasoning to combine conclusions. This is what our notion of cut-absorption (introduced later in Definition 4.5) formalises: we show that cut elimination essentially amounts to the fact that - in the corresponding 
Hilbert system - each valid formula is a consequence of a at most one rule conclusion with provable premise.

We now set the stage for sequent systems that we are going to address in the remainder of the paper. As we are dealing with extensions of classical propositional logic, it suffices to work with a right-handed calculus. Our calculus is equipped with explicit negation, and therefore precisely dual to modal tableau calculi [9] that serve as the usual basis for syntactically determining the complexity of the satisfiability problem.

The notion of derivability in the sequent calculus associated with a rule set $\mathrm{R}$ is formulated parametric in terms of a set $\mathbf{X}$ of additional rules that will later be instantiated with relativised versions of cut, weakening, contraction and inversion.

Definition 3.7. Suppose $R$ and $X$ are sets of $\Lambda$-rules. The set of $G R+X$-derivable sequents in the Gentzen-system given by $\mathrm{R}$ is the least set of sequents that

- contains $A, \neg A, \Gamma$ for all sequents $\Gamma \in \mathrm{S}(\Lambda)$ and formulas $A \in \mathcal{F}(\Lambda)$

- contains $\neg \perp, \Gamma$ for all $\Gamma \in \mathrm{S}(\Lambda)$

- is closed under instances of the rule schemas

$$
(\neg \wedge) \frac{\Gamma, \neg A, \neg B}{\Gamma, \neg(A \wedge B)} \quad(\wedge) \frac{\Gamma, A \quad \Gamma, B}{\Gamma, A \wedge B} \quad(\neg) \frac{\Gamma, A}{\Gamma, \neg \neg A}
$$

where $A \in \mathcal{F}(\Lambda)$ ranges over formulas and $\Gamma \subseteq \mathcal{F}(\Lambda)$ over multisets of formulas. We call the above rules the propositional rules and the formula occurring in the conclusion but not in $\Gamma$ principal in the respective rule.

- is closed under the rules in $\mathrm{R} \cup \mathrm{X}$, i.e. it contains $\Gamma_{0}$ whenever it contains $\Gamma_{1}, \ldots, \Gamma_{n}$ and $\frac{\Gamma_{1} \ldots \Gamma_{n}}{\Gamma_{0}} \in \mathrm{R} \cup \mathrm{X}$.

We write $G R+X \vdash \Gamma$ if $\Gamma$ can be derived in this way and GR $\vdash \Gamma$ if $X=\emptyset$. As for Hilbertstyle calculi (Definition 3.4), we call a rule set $\mathrm{R}^{\prime}$ admissible in GR in case GR $\vdash \Gamma \Longleftrightarrow$ $\mathrm{G}\left(\mathrm{R} \cup \mathrm{R}^{\prime}\right) \vdash \Gamma$ for all $\Gamma \in \mathrm{S}(\Lambda)$.

The set $X$ of extra rules will later be instantiated with a relativised version of the cut rule and additional axioms that locally capture the effect of weakening, contraction and inversion, applied to rule premises. This allows to formulate local conditions for the admissibility of cut that can be checked on a per-rule basis.

Many other formulations of sequent systems only permit axioms of the form $\Gamma, p, \neg p$ where $p \in V$ is a propositional atom. The reason for being more liberal here is that this makes it easier to prove admissibility of uniform substitution, at the expense of losing depthpreserving admissibility of structural rules. We come back to this matter in Remark 4.4. The following proposition is readily established by an induction on the provability predicate $\mathrm{HR} \vdash$.

Proposition 3.8. Suppose $\Gamma \in \mathrm{S}(\Lambda)$ is a sequent. Then $\mathrm{HR} \vdash \bigvee \Gamma$ if $\mathrm{GR} \vdash \Gamma$.

The remainder of the paper is concerned with the converse of the above proposition, which relies on specific properties of the rule set $R$.

\section{Generic Modal Cut Elimination}

In order to establish the converse of Proposition 3.8 we need to establish that the cut rule is admissible in the Gentzen system GR defined by the ruleset R. Clearly, we cannot expect that cut elimination holds in general: it is well known (and easy to check) 
that the sequent system arising from the rule set consisting of all instances of (N) and (D), presented in Example 3.2 does not enjoy cut elimination. In other words, we have to look for constructions that allow us to transform a given rule set into one for which cut elimination holds. The main result of our analysis is that cut elimination holds if the rule set under consideration satisfies four crucial requirements that are local in the sense that they can be checked on a per-rule basis without the need of carrying out a fully-fledged cut-elimination proof: absorption of weakening, contraction, inversion and cut.

The first three properties can be checked for each rule individually and amount to the admissibility of the respective principle, and the last requirement amounts to the possibility of eliminating cut between a pair of rule conclusions. We emphasise that these properties can be checked locally for the modal rules, and cut elimination will follow automatically. It is not particularly surprising that cut elimination holds under these assumptions. However, isolating the four conditions above provides us with means to convert a modal Hilbert system into an equivalent cut-free sequent calculus. We now introduce relativised versions of the structural rules that will be the main tool in the proof of cut elimination. This can be seen as permutability of structural rules: every derivation of $\Gamma$ from premises $\Gamma_{1}, \ldots, \Gamma_{n}$ that ends in weakening, inversion or contraction is applied can be replaced by a derivation of $\Gamma$ where weakening, inversion and contraction is only applied to the premises $\Gamma_{1}, \ldots, \Gamma_{n}$.

Definition 4.1. Suppose $\Gamma$ is a $\Lambda$-sequent and let $\mathrm{A}(\Gamma)$ consist of the axioms

- $\Gamma, A$ for all $A \in \mathcal{F}(\Lambda)$

- $\Delta, A$ if $\Gamma=\Delta, A, A$ for some $\Delta \in \mathrm{S}(\Lambda), A \in \mathcal{F}(\Lambda)$

- $\Delta, A$ if $\Gamma=\Delta, \neg \neg A$ for some $\Delta \in \mathrm{S}(\Lambda), A \in \mathcal{F}(\Lambda)$

- $\Delta, \neg A_{1}, \neg A_{2}$ if $\Gamma=\Delta, \neg\left(A_{1} \wedge A_{2}\right)$ for some $\Delta \in \mathrm{S}(\Lambda), A_{1}, A_{2} \in \mathcal{F}(\Lambda)$

- $\Delta, A_{i}$ for $i=1,2$ if $\Gamma=\Delta,\left(A_{1} \wedge A_{2}\right)$ for some $\Delta \in \mathrm{S}(\Lambda), A_{1}, A_{2} \in \mathcal{F}(\Lambda)$

We say that a rule set $\mathrm{R}$ absorbs the structural rules if

$$
\mathrm{GR}+\mathrm{A}\left(\Gamma_{1}\right) \cup \cdots \cup \mathrm{A}\left(\Gamma_{n}\right) \vdash \Gamma
$$

for all $\frac{\Gamma_{1} \ldots \Gamma_{n}}{\Gamma_{0}} \in \mathrm{R}$ and all $\Gamma \in \mathrm{A}\left(\Gamma_{0}\right)$.

In other words, a deduction step that applies weakening, contraction or inversion to a rule conclusion can be replaced by a (possibly different) rule where the corresponding structural rules are applied to the premises. We discuss a number of standard examples before stating that absorption of the structural rules implies their admissibility.

Example 4.2. The rule sets containing all instances of either of the following rule schemas $(\mathrm{K}),(\mathrm{T})$, and (K4), respectively,

$$
\text { (K) } \frac{\neg A_{1}, \ldots, \neg A_{n}, A_{0}}{\neg \square A_{1}, \ldots, \neg \square A_{n}, \square A_{0}, \Gamma} \quad \text { (T) } \frac{\neg A, \neg \square A, \Gamma}{\neg \square A, \Gamma} \quad \text { (K4) } \frac{\neg A_{1}, \neg \square A_{1}, \ldots, \neg A_{n}, \neg \square A_{n}, B}{\neg \square A_{1}, \ldots, \neg \square A_{n}, \square B, \Gamma}
$$

absorb the structural rules. We note that $(\mathrm{K})$ absorbs weakening due to the presence of $\Gamma$ in the conclusion, and the absorption of contraction in (T) and (K4) is a consequence of the presence of the negated $\square$-formulas in the premise. The absorption of inversion in a consequence of the weakening context $\Gamma$ in $(\mathrm{K})$ and $(\mathrm{K} 4)$ and implied by duplicating the context $\Gamma$ in $(\mathrm{T})$. On the other hand, the rule sets defined by

$$
\frac{\neg A_{1}, \ldots, \neg A_{n}, A_{0}}{\neg \square A_{1}, \ldots, \neg \square A_{n}, \square A_{0}} \quad \frac{\neg A, \Gamma}{\neg \square A, \Gamma}
$$

fail to absorb the structural rules: the rule on the left fails to absorb weakening, whereas the right-hand rule does not absorb contraction. 
It should be intuitively clear that absorption of structural rules implies their admissibility, which we establish next.

Proposition 4.3. Suppose R absorbs the structural rules. Then all instances of the rule schemas of weakening, contraction and inversion

$$
\frac{\Gamma}{\Gamma, A} \quad \frac{\Gamma, A, A}{\Gamma, A} \quad \frac{\Gamma, \neg \neg A}{\Gamma, A} \quad \frac{\Gamma, \neg\left(A_{1} \wedge A_{2}\right)}{\Gamma, \neg A_{1}, \neg A_{2}} \quad \frac{\Gamma, A_{1} \wedge A_{2}}{\Gamma, A_{i}}(i=1,2)
$$

where $\Gamma \in \mathrm{S}(\Lambda)$ and $A, A_{1}, A_{2} \in \mathcal{F}(\Lambda)$ are admissible in $\mathrm{GR}$.

Proof. Standard induction on proofs in GR where the case of propositional rules is standard and the inductive case for modal rules immediately follows from absorption.

\section{Remark 4.4.}

(1) The main purpose for introducing the notion of absorption of structural rules (Definition 4.1) is to have a handy criterion that guarantees admissibility of the structural rules (Proposition 4.3). Our definition offers a compromise between generality and simplicity. In essence, a rule set absorbs structural rules if an application of weakening, contraction or inversion can be pushed up one level of the proof tree. A weaker version of Definition 4.1 would require that an application of weakening, contraction or inversion to a rule conclusion can be replaced by a sequence of deduction steps where the structural rule in question can not only be applied to the premises of the rule, but also freely anywhere else, provided that these additional applications are smaller in a well-founded ordering. However, we are presently not aware of any examples where this extra generality would be necessary.

(2) In many sequent systems, the statement of Proposition 4.3 can be strengthened to say that weakening, contraction and inversion are depth-preserving admissible, i.e. does not increase the height of the proof tree. This is in general false for the systems considered here as axioms are of the form $A, \neg A, \Gamma$ for $A \in \mathcal{F}(\Lambda)$ and, for instance, $(A \wedge B), \neg(A \wedge B)$ is derivable with a proof of height one (being an axiom), but, e.g. $A \wedge B, \neg A, \neg B$ cannot be established by a proof of depth one (not being an axiom). It is easy to see that weakening, inversion and contraction are in fact depth-preserving admissible if only atomic axioms of the form $p, \neg p, \Gamma$ are allowed, for $p \in V$ a propositional variable. The more general form of axioms adopted in this paper allows us to simplify many constructions as we do not have to consider a congruence rule explicitly which would allow us to prove (rather than to assume as axioms) sequents of the form $\square A, \neg \square A, \Gamma$.

Having dealt with the structural rules, we now address our main concern: the admissibility of the cut rule. In contrast to the absorption of structural rules, we need one additional degree of freedom in that we need to allow ourselves to apply cut to a structurally smaller formula.

Definition 4.5. The size of a formula $A \in \mathcal{F}(\Lambda)$ is given inductively by $\operatorname{size}(p)=\operatorname{size}(\perp)=$ 1 , size $(A \wedge B)=1+\operatorname{size}(A)+\operatorname{size}(B), \operatorname{size}(\neg A)=1+\operatorname{size}(A)$ and, for the modal case, $\operatorname{size}\left(\varnothing\left(A_{1}, \ldots, A_{n}\right)\right)=1+\operatorname{size}\left(A_{1}\right)+\cdots+\operatorname{size}\left(A_{n}\right)$.

A ruleset $\mathrm{R}$ absorbs cut, if for all rules $\left(r_{1}\right) \frac{\Gamma_{1} \ldots \Gamma_{n}}{A, \Gamma_{0}},\left(r_{2}\right) \frac{\Delta_{1} \ldots \Delta_{k}}{\neg A, \Delta_{0}} \in \mathrm{R}$

$$
\mathrm{GR}+\operatorname{Cut}\left(A, r_{1}, r_{2}\right) \vdash \Gamma_{0}, \Delta_{0}
$$


where Cut $\left(A, r_{1}, r_{2}\right)$ consists of all instances of the rule schemas

$$
\frac{\Gamma, C \quad \Delta, \neg C}{\Gamma, \Delta} \quad \frac{\Gamma}{\Gamma, A} \quad \frac{\Gamma, A, A}{\Gamma, A} \quad \frac{\Gamma, \neg \neg A}{\Gamma, A} \quad \frac{\Gamma, \neg\left(A_{1} \wedge A_{2}\right)}{\Gamma, \neg A_{1}, \neg A_{2}} \quad \frac{\Gamma, A_{1} \wedge A_{2}}{\Gamma, A_{i}}
$$

where $\operatorname{size}(C)<\operatorname{size}(A)$ in the leftmost rule and $i=1,2$ in the rightmost schema, together with the axioms $\Gamma_{1}, \ldots, \Gamma_{n}, \Delta_{1}, \ldots, \Delta_{k}$ and all sequents of the form $\Gamma, \Delta$ where $\Gamma, \Delta \in \mathrm{S}(\Lambda)$ and, for some $B \in \mathcal{F}(\Lambda)$,

- $\Gamma, B$ and $\Delta, \neg B \in\left\{\Gamma_{1}, \ldots, \Gamma_{n}, \Delta_{1}, \ldots, \Delta_{k}\right\}$, or

- $\Gamma, B=\Gamma_{0}, A$ and $\Delta, \neg B \in\left\{\Delta_{1}, \ldots, \Delta_{k}\right\}$, or

- $\Gamma, B=\Delta_{0}, \neg A$ and $\Delta, \neg B \in\left\{\Gamma_{1}, \ldots, \Gamma_{n}\right\}$.

A rule set that absorbs structural rules and the cut rule is called absorbing.

The intuition behind the above definition is similar to that of absorption of structural rules, but we have two additional degrees of freedom: we can not only apply the cut rule to rule premises, but we can moreover freely use both cut on structurally smaller formulas and the structural rules. This allows us to use the standard argument, a double induction on the structure of the cut formula and the size of the proof tree, to establish cut elimination. This is carried out in the proof of the next theorem.

Theorem 4.6. Suppose $\mathrm{R}$ is absorbing. Then the cut rule

$$
\frac{\Gamma, A \quad \Delta, \neg A}{\Gamma, \Delta}
$$

is admissible in $\mathrm{GR}$.

Proof. We use Gentzen's classical method and proceed by a double induction on the size of the cut formula and the size of the proof tree. That is, we prove the statement

$$
\forall A \in \mathcal{F}(\Lambda) \forall n \in \omega\left(n=n_{1}+n_{2} \& \vdash_{n_{1}} \Gamma, A \& \vdash_{n_{2}} \Delta, \neg A \Longrightarrow \vdash \Gamma, \Delta\right)
$$

by induction on $\operatorname{size}(A)$ where, in the inductive step, we use a side induction on the size of proof trees, as indicated by the subscript of the entailment sign. Formally, the relation $\vdash_{n}$ is defined inductively by $\vdash_{1} \Gamma, A, \neg A$ and

$$
\frac{\vdash_{n} A}{\vdash_{n+1} \neg \neg A} \quad \frac{\vdash_{n} \Gamma, \neg A, \neg B}{\vdash_{n+1} \Gamma, \neg(A \wedge B)} \quad \frac{\vdash_{n} \Gamma, A \quad \vdash_{k} \Gamma, B}{\vdash_{n+k+1} \Gamma, A \wedge B} \quad \frac{\vdash_{n_{1}} F_{1} \ldots \vdash_{n_{k}} F_{k}}{\vdash_{n_{1}+\cdots+n_{k}+1} F_{0}}
$$

where, in the last rule, $\frac{F_{1} \ldots F_{k}}{F_{0}} \in \mathrm{R}$. We may inductively assume that the statement holds for all cut formulas $C<A$ and to prove the statement for $A$ we have to consider the following cases:

(1) cuts that arise between two rule conclusions

(2) cuts that arise between a rule conclusion and the conclusion of a propositional rule or axiom

(3) cuts that arise between two propositional rules.

We start with item (1), which follows directly from the fact that $\mathrm{R}$ absorbs cut. In more detail, suppose that $\frac{F_{1} \ldots F_{k}}{F_{0}, A}$ and $\frac{G_{1} \ldots G_{k}}{G_{0}, \neg A} \in \mathrm{R}$ and $\vdash_{n_{i}} F_{i}(i=1, \ldots, k)$ and $\vdash_{m_{j}} G_{j}$ for $j=1, \ldots, l$. As R absorbs cut, we have that $F_{0}, G_{0}$ is derivable using cuts on formulas $<A$ from the additional assumptions $\Gamma, \Delta$ provided that for some $D \in \mathcal{F}(\Lambda)$ we have that both $\Gamma, D$ and $\Delta, \neg D$ are among the $F_{1}, \ldots, F_{k}, G_{1}, \ldots, G_{l}$. In case $\Gamma, D=\Delta, \neg D$ we have that $\Gamma \subseteq \Gamma, \Delta$ and $\vdash \Gamma, \Delta$ as weakening is admissible in GR. Assuming that $\vdash_{x} \Gamma, D$ and $\vdash_{y} \Delta, \neg D$ for $\Gamma, D \neq \Delta, \neg D$ we have that $x+y<2+\sum_{i} n_{i}+\sum_{j} m_{j}$ and hence $\vdash \Gamma, \Delta$ by 
(inner) induction hypothesis. The fact that - in the deduction of $F_{0}, G_{0}$ - we may also have to use cuts on formulas $<A$ is discharged by the outer induction hypothesis and possible uses of weakening, contraction and inversion are admissible by Proposition 4.3.

As regards item (2) we only discuss a subset of the cases that showcase the need for contraction, weakening and inversion to be admissible. For the whole discussion, suppose that $\frac{F_{1} \ldots F_{k}}{F_{0}} \in \mathrm{R}$ and $\vdash_{n_{i}} F_{i}$ for $i=1, \ldots, k$.

- Suppose that $F_{0}=F_{0}^{\prime}, A$ and $G_{0}, \neg A$ is an axiom. In case $A \in G_{0}$ we have that $F_{0}=$ $F_{0}^{\prime}, A \subseteq F_{0}^{\prime}, G_{0}$ and $\vdash F_{0}^{\prime}, G_{0}$ follows from $\vdash F_{0}^{\prime}, A$ as $\mathrm{GR}$ admits weakening. In case $\neg A \notin G_{0}$ we have that $G_{0}$ is an axiom, and hence so is $G_{0}, F_{0}^{\prime}$.

- Suppose that $F_{0}=F_{0}^{\prime}, A$ and $\neg A, G_{0}$ has been derived using $(\neg \wedge)$. We have to discuss two cases, depending on whether or not $\neg A$ is principal in the application of $(\neg \wedge)$.

Case $A=A^{\prime} \wedge B^{\prime}$ and $\vdash_{m} \neg A^{\prime}, \neg B^{\prime}, G_{0}$ so that $\vdash_{m+1} \neg A, G_{0}$. As $\mathrm{R}$ absorbs structural rules, we have that $\mathrm{GR} \vdash F_{0}^{\prime}, A$ and $\mathrm{GR} \vdash F_{0}^{\prime}, B$. As cuts on $A^{\prime}$ and $B^{\prime}$ can be eliminated by induction hypothesis, we have GR $\vdash F_{0}^{\prime}, F_{0}^{\prime}, G_{0}$ and therefore GR $\vdash F_{0}^{\prime}, G_{0}$ as GR admits contraction.

Case $\vdash_{m} \neg C, \neg D, \neg A, G_{0}$ so that $\vdash_{m+1} \neg(C \wedge D), \neg A, G_{0}$. As $m+1+\sum_{i=1}^{k} n_{i}<m+1+$ $1+\sum_{i=1}^{k} n_{i}$ we may apply the inner induction hypothesis to conclude $\vdash \neg C, \neg D, G_{0}, F_{0}^{\prime}$ and applying $(\neg \wedge)$ gives $\vdash F_{0}^{\prime}, \neg(C \wedge D), G_{0}$.

All the other cases follow exactly the same pattern. We now focus on item (3), that is, we show how cuts between the conclusions of propositional rules and axioms can be eliminated. This is mostly standard and again we only discuss a subset of the cases. Suppose that $\vdash_{n} F_{0}, A$ and $\vdash_{m} G_{0}, \neg A$.

- If both $F_{0}, A$ and $G_{0}, \neg A$ are axioms, then so is $F_{0}, G_{0}$.

- Suppose that $F_{0}, A$ has been derived using $(\wedge)$ and $G_{0}, \neg A$ has been derived using $(\neg \wedge)$. We distinguish four cases depending on whether or not $A$ is principal in the application of $(\wedge)$ or $(\neg \wedge)$.

Case $A=A^{\prime} \wedge B^{\prime}$ and $\vdash_{n_{0}} F_{0}, A, \vdash_{n_{1}} F_{0}, B^{\prime}$ so that $n=n_{0}+n_{1}+1$ and $\vdash_{n} A \wedge B, F_{0}$. If $A$ is principal in the application of $(\neg \wedge)$, we have that $\vdash_{m-1} G_{0}, \neg A^{\prime}, \neg B^{\prime}$. By (outer) induction hypothesis, cuts on $A^{\prime}$ and $B^{\prime}$ can be eliminated so that we have $\vdash F_{0}, F_{0}, G_{0}$ and it follows from closure under contraction that $\vdash F_{0}, G_{0}$.

If $A$ is not principal in the application of $(\neg \wedge)$ we have that $\vdash_{m-1} \neg C, \neg D, \neg\left(A^{\prime} \wedge B^{\prime}\right), G_{0}^{\prime}$ so that $G_{0}=\neg(C \wedge D), G_{0}^{\prime}$ and $\vdash_{m} \neg(A \wedge B), G_{0}^{\prime}$. As $\vdash_{n} F_{0}, A$ and $\vdash_{m-1} \neg C, \neg D, \neg A, G_{0}^{\prime}$ and $n+(m-1)<n+m$ we can apply the inner induction hypothesis to eliminate the cut on $A$ so that $\vdash F_{0}, \neg C, \neg D, G_{0}^{\prime}$ and applying $(\neg \wedge)$ gives $\vdash F_{0}, \neg(C \wedge D), G_{0}^{\prime}=F_{0}, G_{0}$ as required. The two cases where $A$ is not principal in the application of $(\neg \wedge)$ follow exactly the same pattern.

The remaining cases of cuts between propositional rules and axioms are entirely analogous, and therefore omitted.

We illustrate the preceding theorem by using it to derive the well-known fact that cutelimination holds for the modal logics $\mathrm{K}, \mathrm{K} 4$ and $\mathrm{T}$ and use it to derive cut-elimination for various conditional logics in Section 6 .

Example 4.7. The rule sets $\mathrm{K}, \mathrm{K} 4$ and $\mathrm{T}$ are absorbing. We have already seen that they absorb weakening, contraction and inversion in Example 4.2 so everything that remains to be seen is that they also absorb cut. For $(K)$, we need to apply cut to a formula of smaller 
size. For the two instances

$$
\left(r_{1}\right) \frac{\neg A_{1}, \ldots, \neg A_{n}, A_{0}}{\neg \square A_{1}, \ldots, \neg \square A_{n}, \square A_{0}, \Gamma} \quad\left(r_{2}\right) \frac{\neg B_{1}, \ldots, \neg B_{k}, B_{0}}{\neg \square B_{1}, \ldots, \neg \square B_{k}, \square B_{0}, \Delta}
$$

we need to consider, up to symmetry, the cases $A_{i}=B_{0}, \square A_{i} \in \Delta$ and $\neg \square A_{0} \in \Delta$, for $i=1, \ldots, n$. Here, we only treat the first case for $i=1$ where we have to show that $\neg \square A_{2}, \ldots, \neg \square A_{n}, \square A_{0}, \neg \square B_{1}, \ldots, \neg \square B_{k}, \Gamma, \Delta$ is derivable from GR $+\operatorname{Cut}\left(\square A_{1}, r_{1}, r_{2}\right)$, which follows as the latter system allows us to apply cut on $A_{1}=B_{0}$. The case $\square A_{i} \in \Delta$ and $\neg \square A_{0} \in \Delta$ are straight forward.

The argument to show that (K4) is absorbing is similar, and uses an additional (admissible) instance of cut on a formula of smaller size and contraction. For $(\mathrm{T})$ we only consider instances of cut between two conclusions of

$$
\left(r_{1}\right) \frac{\neg A, \neg \square A, \Gamma}{\neg \square A, \Gamma} \quad\left(r_{2}\right) \frac{\neg B, \neg \square B, \Delta}{\neg \square B, \Delta}
$$

of the T-rule. We only demonstrate the case $\square A \in \Delta$. In this case, $\Delta=\Delta^{\prime}, \square A$ and we have to show that $\neg \square B, \Gamma, \Delta^{\prime}$ can be derived in $\operatorname{Cut}\left(\square A, r_{1}, r_{2}\right)$. The latter system allows us to cut $\neg \square A$ between the conclusion of ( $\mathrm{T}$ ) on the left and the premise of the right hand rule, i.e., we have that $\left.\operatorname{Cut}\left(\square A, r_{1}, r_{2}\right) \vdash \neg B, \neg \square B, \Gamma, \Delta^{\prime}\right)$ and an application of ( $\left.\mathrm{T}\right)$ now gives derivability of $\neg \square B, \Gamma, \Delta^{\prime}$.

\section{Equivalence of Hilbert and Gentzen Systems}

We now investigate the relationship between provability in a Hilbert-system and provability in the associated Gentzen system. We note the following standard lemmas that we will use later on.

Lemma 5.1. Suppose that $\Lambda$ is a modal similarity type and $\mathrm{R}$ is a set of $\Lambda$-rules.

(1) Let $A \in \mathcal{F}(\Lambda)$ be a propositional tautology. Then $\mathrm{GR} \vdash A$.

(2) Let $\mathrm{R}$ be closed under substitution and $\Gamma \in \mathrm{S}(\Lambda)$. Then $\mathrm{GR} \vdash \Gamma \sigma$ whenever $\mathrm{GR} \vdash \Gamma$.

Remark 5.2. Being able to prove the previous lemma is the main reason for formulating axioms as $A, \neg A, \Gamma$ where $A \in \mathcal{F}(\Lambda)$ rather than $p, \neg p, \Gamma$. Both formulations are equivalent if the modal congruence rule

$$
\frac{A_{1} \leftrightarrow A_{1}^{\prime} \quad \ldots \quad A_{n} \leftrightarrow A_{n}^{\prime}}{\varnothing\left(A_{1}, \ldots, A_{n}\right) \rightarrow \varnothing\left(A_{1}^{\prime}, \ldots, A_{n}^{\prime}\right)}
$$

is admissible. However, Lemma 5.1 can be proved without the assumption that congruence is admissible using axioms of the form $A, \neg A, \Gamma$.

Theorem 5.3. Suppose $\mathrm{R}$ is absorbing and substitution closed. Then $\mathrm{GR} \vdash \Gamma \Longleftrightarrow \mathrm{HR} \vdash$ $\bigvee \Gamma$ for all $\Gamma \in \mathrm{S}(\Lambda)$.

Sketch. We only need to show the direction from right to left. Inductively assume that $\mathrm{HR} \vdash \bigvee \Gamma$ for $\Gamma \in \mathrm{S}(\Lambda)$. By Proposition 3.8 we have that there are rules $\Theta_{i} / \Gamma_{i}$ and substitutions $\sigma_{i}, i=1, \ldots, n$ such that

- $\mathrm{HR} \vdash \Delta \sigma_{i}$ whenever $\Delta \in \Theta_{i}(i=1, \ldots, n)$

- $\left\{\bigvee \Gamma_{1} \sigma_{1}, \ldots, \bigvee \Gamma_{n} \sigma_{n}\right\} \vdash_{\mathrm{PL}} \bigvee \Gamma$. 
By induction hypothesis, GR $\vdash \Delta \sigma_{i}$ for all $i=1, \ldots, n$ and $\Delta \in \Theta_{i}$. By Lemma 5.1 we have

$$
\mathrm{GR} \vdash \bigvee \Gamma_{1} \sigma_{1} \wedge \cdots \wedge \bigvee \Gamma_{n} \sigma_{n} \rightarrow \bigvee \Gamma
$$

The claim follows by applying cut, contraction and inversion.

The construction of an absorbing rule set from a given set of axioms and rules essentially boils down to adding the missing instances of cut, weakening, contraction and inversion to a given rule set. The soundness of this process is witnessed by the following two trivial lemmas (both of which rest on the fact that HR incorporates full propositional reasoning). We use these to derive an absorbing rule set for $K$ in the present section, and to establish cut-elimination for a large range of conditional logics in the next section.

Lemma 5.4. Suppose $\Gamma_{1}, \ldots, \Gamma_{n} / \neg A, \Gamma_{0}$ and $\Delta_{1}, \ldots, \Delta_{k} / A, \Delta_{0} \in \mathrm{R}$. Then the rule $\Gamma_{1}, \ldots, \Gamma_{n}, \Delta_{1}, \ldots, \Delta_{k} / \Gamma_{0}, \Delta_{0}$ is admissible in $\mathrm{HR}$.

The same applies to instances of the structural rules of weakening, contraction and inversion. As we wish to extend the rule set while leaving the provability predicate in the Hilbert calculus unchanged, the following formulation is handy for our purposes - in particular it implies that we can freely use structural rules both in the premise and conclusion.

Lemma 5.5. Let $\Gamma_{1}, \ldots, \Gamma_{n} / \Gamma_{0} \in \mathrm{R}$. If $\Delta_{0}, \ldots, \Delta_{k} \in \mathrm{S}(\Lambda)$ and both

$$
\left\{\bigvee \Delta_{1}, \ldots, \bigvee \Delta_{k}\right\} \vdash_{\mathrm{PL}} \bigvee \Gamma_{i}(1 \leq i \leq n) \text { and } \bigvee \Gamma_{0} \vdash_{\mathrm{PL}} \bigvee \Delta_{0}
$$

then the rule $\Delta_{1}, \ldots, \Delta_{k} / \Delta_{0}$ is admissible in $\mathrm{HR}$.

This gives us a recipe for constructing rule sets that absorb contraction and cut: simply add more rules according to the lemmas above. This will not change the notion of provability in the Hilbert system, but when this process terminates, the ensuing rule set will be absorbing and gives rise to a cut free sequent calculus.

Example 5.6 (Modal Logic $K$ ). In a Hilbert-style calculus, the axiomatisation of $K$ is usually described in terms of the distribution axiom (which we view as a rule with empty premise) and the necessitation rule:

$$
\text { (D) } \square(A \rightarrow B) \rightarrow \square A \rightarrow \square B \quad \text { (N) } \frac{A}{\square A}
$$

We first apply Lemma 5.4 to break the propositional connectives in the distribution axiom. We have that the axiom $\neg \square(A \rightarrow B), \neg \square A, \square B$ is admissible by Lemma 5.5, and applying Lemma 5.4 to this axiom and the instance $A \rightarrow B / \square(A \rightarrow B)$ of the necessitation rule gives admissibility of the all instances of

$$
\frac{\neg A, B}{\neg \square A, \square B}
$$

with the help of (admissible) propositional reasoning in the premise. The same procedure, applied to the instances

$$
\frac{\neg A, B \rightarrow C}{\neg \square A, \square(B \rightarrow C)} \quad \neg \square(B \rightarrow C), \neg \square B, \square C
$$

gives admissibility of the left hand rule below,

$$
\frac{\neg A, \neg B, C}{\neg \square A, \neg \square B, \square C} \quad \frac{\neg A_{1}, \ldots, \neg A_{n}, A_{0}}{\neg \square A_{1}, \ldots, \neg \square A_{n}, \square A_{0}, \Gamma}
$$


and continuing in this way and absorbing weakening, we obtain admissibility of the rule on the right, where $\Gamma \in \mathrm{S}(\Lambda)$ is an arbitrary context. We have shown previously that this rule set is absorbing, and it is easy to see that it is equivalent to the rule set consisting of all instances of $(\mathrm{N})$ and $(\mathrm{D})$.

\section{Applications: Sequent Calculi for Conditional Logics}

After having seen how the construction of absorbing rule sets gives rise to cut-elimination for a number of well-studied normal modal logics, in this section we construct a cut-free sequent calculus for a number of conditional logics.

Conditional logics [3] are extensions of propositional logic by a non-monotonic conditional $A \Rightarrow B$, read as " $B$ holds under the condition that $A$ ". Formulas of the form $A \Rightarrow B$ or $\neg(A \Rightarrow B)$ are called conditional literals, and in such a conditional literal, we refer to $A$ as the (conditional) antecedent and to $B$ as the (conditional) consequent. The conditional implication is non-monotonic in general, i.e. the validity of $A \Rightarrow B$ does not imply that $(A \wedge C) \Rightarrow B$ is also a valid statement.

Axiomatically, the first argument $A$ of the conditional operation $A \Rightarrow B$ behaves like the $\square$ in neighbourhood frames and only supports replacement of equivalents, whereas the second argument $B$ obeys the rules of $K$. We recall from Example 3.2 (see also Figure 2) that CK is axiomatised by the rules (RCEA) and (RCK) that we augment with a subset of (ID), (MP) and (CEM). For each system, we apply Lemma 5.5 and Lemma 5.4 to the given rule sets repeatedly to generate new rules that are automatically sound over the original Hilbert system. This procedure leads to the rules summarised in Figure 3 where we have used the following notational shorthand to express the equivalences in the premise of $\mathrm{CK}$ :

Notation 6.1. If $A_{0}, \ldots, A_{n} \in \mathcal{F}(\Lambda)$ are conditional formulas, we write $A_{0}=\cdots=A_{n}$ for the sequence of sequents consisting of $\neg A_{0}, A_{i}$ and $\neg A_{i}, A_{0}$ for all $1 \leq i \leq n$.

We now discuss the arising system in detail, and start with those not containing (CEM) and then proceed to add (CEM) as an additional principle. For each system, we show cut-free completeness and develop the format of the respective rules as we go along. In summary, we obtain the following cut-free sequent calculi for extensions of (CK) summarised in Figure 4.

6.1. Cut Elimination for Extensions of CK without CEM. We first treat extensions of the basic conditional logic CK with axioms ID and MP, but not including CEM and discuss CEM later, as the effect of adding CEM leads to a more general form of the CK rule.

If we absorb cuts using Lemmas 5.4 and 5.5 we see that all instances of

$$
\left(\mathrm{CK}_{g}\right) \frac{A_{0}=\cdots=A_{n} \quad \neg B_{1}, \ldots, \neg B_{n}, B_{0}}{\neg\left(A_{1} \Rightarrow B_{1}\right), \ldots, \neg\left(A_{n} \Rightarrow B_{n}\right),\left(A_{0} \Rightarrow B_{0}\right), \Gamma}
$$

are admissible in HCK. It is easy to see that the rule set $\mathrm{CK}_{g}$ is actually absorbing:

Theorem 6.2. The rule set $\mathrm{CK}_{g}$ is absorbing and equivalent to $\mathrm{CK}$. As a consequence, $\mathrm{GCK}_{g}$ has cut-elimination and $\mathrm{GCK}_{g} \vdash A$ iff $\mathrm{HCK} \vdash A$ whenever $A \in \mathcal{F}(\Lambda)$.

Proof. Using Lemmas 5.4 and Lemma 5.5 it is immediate that the rule set $\mathrm{CK}_{g}$ is admissible in $\mathrm{HCK}$. The argument that shows that $\mathrm{CK}_{g}$ is absorbing is analogous to that for the modal logic $K$ (Example 4.7), and the result follows from Theorem 5.3 , 


$$
\begin{gathered}
\left(\mathrm{CK}_{g}\right) \frac{A_{0}=\cdots=A_{n} \quad \neg B_{1}, \ldots, \neg B_{n}, B_{0}}{\neg\left(A_{1} \Rightarrow B_{1}\right), \ldots, \neg\left(A_{n} \Rightarrow B_{n}\right),\left(A_{0} \Rightarrow B_{0}\right), \Gamma} \\
\left(\mathrm{CKID}_{g}\right) \frac{A_{0}=\cdots=A_{n} \quad \neg A_{0}, \neg B_{1}, \ldots, \neg B_{n}, B_{0}}{\neg\left(A_{1} \Rightarrow B_{1}\right), \ldots, \neg\left(A_{n} \Rightarrow B_{n}\right),\left(A_{0} \Rightarrow B_{0}\right), \Gamma} \\
\left(\mathrm{MP}_{g}\right) \frac{A, \neg(A \Rightarrow B), \Gamma \quad \neg B, \neg(A \Rightarrow B), \Gamma}{\neg(A \Rightarrow B), \Gamma} \\
\left(\mathrm{CKCEM}_{g}\right) \frac{A_{0}=\ldots=A_{n} \quad B_{0}, \ldots, B_{j}, \neg B_{j+1}, \neg B_{n}}{\left(A_{0} \Rightarrow B_{0}\right), \ldots,\left(A_{j} \Rightarrow B_{j}\right), \neg\left(A_{j+1} \Rightarrow B_{j+1}\right), \ldots, \neg\left(A_{n} \Rightarrow B_{n}\right), \Gamma} \quad(0 \leq j \leq n) \\
\left(\mathrm{CKCEMID}_{g}\right) \frac{A_{0}=\ldots=A_{n} \quad \neg A_{0}, B_{0}, \ldots, B_{j}, \neg B_{j+1}, \neg B_{n}}{\left(A_{0} \Rightarrow B_{0}\right), \ldots,\left(A_{j} \Rightarrow B_{j}\right), \neg\left(A_{j+1} \Rightarrow B_{j+1}\right), \ldots, \neg\left(A_{n} \Rightarrow B_{n}\right), \Gamma} \\
\left(\mathrm{MPCEM}_{g}\right) \frac{A,(A \Rightarrow B), \Gamma \quad B,(A \Rightarrow B), \Gamma}{(A \Rightarrow B), \Gamma}
\end{gathered}
$$

Figure 3: Cut-Free Conditional Sequent Rules

\begin{tabular}{|l|l|l|l|}
\hline Hilbert System & Sequent System & Hilbert System & Sequent System \\
\hline \hline CK & $\mathrm{CK}_{g}$ & CKCEM & CKCEM $_{g}$ \\
\hline CKID & $\mathrm{CKID}_{g}$ & CKCEMID & CKCEMID $_{g}$ \\
\hline CKMP & $\mathrm{CK}_{g}+\mathrm{MP}_{g}$ & CKCEMMP & CKCEM $_{g}+\mathrm{MP}_{g}+\mathrm{MPCEM}_{g}$ \\
\hline CKMPID & $\mathrm{CKID}_{g}+\mathrm{MP}_{g}$ & CKCEMMPID & CKCEMID $_{g}+\mathrm{MP}_{g}+\mathrm{MPCEM}_{g}$ \\
\hline
\end{tabular}

Figure 4: Summary of Cut-Free Sequent Systems

The logic CKID arises form CK by adding the identity axiom $A \Rightarrow A$ to the rule set $\mathrm{CK}_{H}$ that axiomatises standard conditional logic. Applying Lemma 5.4 to the two rule instances

$$
\frac{A=A \quad \neg A, B}{\neg(A \Rightarrow A),(A \Rightarrow B)} \quad \overline{A \Rightarrow A}
$$

yields the (admissible) rule

$$
\frac{\neg A, B}{A \Rightarrow B}
$$

Again applying the same lemma, this time to a general instance of $(\mathrm{CK})$ and the rule that we just derived, that is,

$$
\frac{A_{0}=C=A_{1} \cdots=A_{n} \quad \neg D, \neg B_{1}, \ldots, \neg B_{n}, B_{0}}{\neg(C \rightarrow D), \neg\left(A_{1} \Rightarrow B_{1}\right), \ldots, \neg\left(A_{n} \Rightarrow B_{n}\right)} \quad \frac{\neg C, D}{C \Rightarrow D}
$$

now gives the rule

$$
\left(\mathrm{CKID}_{g}\right) \frac{A_{0}=\cdots=A_{n} \quad \neg A_{0}, \neg B_{1}, \ldots, \neg B_{n}, B_{0}}{\neg\left(A_{1} \Rightarrow B_{1}\right), \ldots, \neg\left(A_{n} \Rightarrow B_{n}\right),\left(A_{0} \Rightarrow B_{0}\right), \Gamma}
$$

that can be again seen to be admissible by Lemma 5.5. It is easy to see that both (CK) and (ID) are derivable under (CKID), and we note that $\left(\mathrm{CKID}_{g}\right)$ is admissible by construction. If we denote the rule set consisting of all instances of CKID by $\mathrm{CKID}_{g}$, we obtain: 
Proposition 6.3. The rule set $\mathrm{CKID}_{g}$ is absorbing and equivalent to CKID.

Proof. It is easy to see that $\mathrm{CKID}_{g}$ absorbs the structural rules, and that CKID is equivalent to $\mathrm{CKID}_{g}$.

To see that $\mathrm{CKID}_{g}$ absorbs cut, we consider two instances of $\left(\mathrm{CKID}_{g}\right)$, say

$$
\left(r_{1}\right) \frac{A_{0}=\cdots=A_{n} \quad \neg B_{1}, \ldots, \neg B_{n}, B_{0}}{\neg\left(A_{1} \Rightarrow B_{1}\right), \ldots, \neg\left(A_{n} \Rightarrow B_{n}\right),\left(A_{0} \Rightarrow B_{0}\right), \Gamma}
$$

and

$$
\left(r_{2}\right) \frac{C_{0}=\cdots=C_{k} \quad \neg D_{1}, \ldots, \neg D_{k}, D_{0}}{\neg\left(C_{1} \Rightarrow D_{1}\right), \ldots, \neg\left(C_{k} \Rightarrow D_{k}\right),\left(C_{0} \Rightarrow D_{0}\right), \Delta}
$$

and assume that the cut happens on $F \in \mathcal{F}(\Lambda)$. The case where $F \in \Gamma, \Delta$ is straightforward, so assume without loss of generality that $F=\left(A_{0} \Rightarrow B_{0}\right)=\left(C_{1} \Rightarrow D_{1}\right)$. By converting the equalities in the premise, and repeatedly applying cut on $A_{0} \equiv C_{1}$ we obtain

$$
\frac{C_{0}, \neg C_{i} \quad A_{0}, \neg A_{i}}{C_{0}, \neg A_{i}} \operatorname{Cut}\left(A_{0} \equiv C_{1}\right) \quad \frac{\neg C_{0}, C_{i} \quad \neg A_{0}, A_{i}}{\neg C_{0}, A_{i}} \operatorname{Cut}\left(A_{0} \equiv C_{i}\right)
$$

so that we obtain the derivability of

$$
\Sigma_{1}=C_{0}=A_{1}=\cdots=A_{n}=C_{2}=\cdots=C_{k}
$$

in $\mathrm{GCKID}_{g}+\operatorname{Cut}\left(F, r_{1}, r_{2}\right)$ (recall Notation 6.1). The derivation

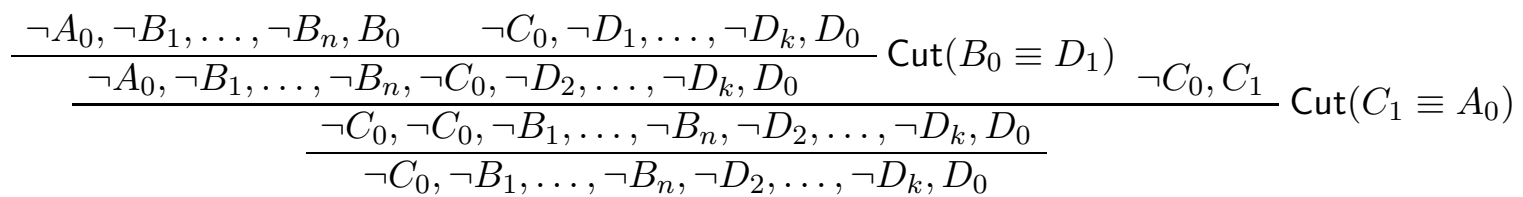

where contraction on $\left(C_{0}\right)$ was applied in the last step, shows that

$$
\Sigma_{2}=\neg C_{0}, \neg B_{1}, \ldots, \neg B_{n}, \neg D_{2}, \ldots, \neg D_{k}, D_{0}
$$

is derivable in $\mathrm{GCKID}_{g}+\operatorname{Cut}\left(F, r_{1}, r_{2}\right)$, and applying $\mathrm{CKID}_{g}$ to $\Sigma_{1}$ and $\Sigma_{2}$ gives (cut-free) derivability of the desired sequent $\neg\left(A_{1} \Rightarrow B_{1}\right), \ldots, \neg\left(A_{n} \Rightarrow B_{n}\right), \neg\left(C_{2} \Rightarrow D_{2}\right), \ldots, \neg\left(C_{k} \Rightarrow\right.$ $\left.D_{k}\right),\left(C_{0} \Rightarrow D_{0}\right), \Gamma, \Delta$. This completes the case distinction on $F$ and hence the proof of the proposition.

Before we move to the next system, we briefly demonstrate the derivation of the identity axiom in $\mathrm{CKID}_{g}$.

Example 6.4. It is easy to say that $\mathrm{GCKID}_{g} \vdash A \Rightarrow A$ for all $A \in \mathcal{F}(\Lambda)$ : we pick $n=0$ to obtain the following instance of $\mathrm{CKID}_{g}$

$$
\frac{\neg A, A}{A \Rightarrow A}
$$

and note that the premise is in fact an axiom.

The logic CKMP arises by augmenting the logic CK with the additional axiom $(A \Rightarrow$ $B) \rightarrow(A \rightarrow B)$. We briefly sketch the construction of the additional axiom that gives rise to the rule $\left(\mathrm{MP}_{g}\right)$ that we will use to establish cut-free completeness.

We consider a cut between an instance of $\left(\mathrm{CK}_{g}\right)$ and $(\mathrm{MP})$, that is, we have the derivation 


$$
\frac{\frac{A_{0}=A_{1} \quad \neg B_{1}, B_{0}}{\neg\left(A_{1} \Rightarrow B_{1}\right),\left(A_{0} \Rightarrow B_{0}\right)} \quad \neg\left(A_{0} \Rightarrow B_{0}\right), A_{0} \rightarrow B_{0}}{\neg\left(A_{1} \Rightarrow B_{1}\right), A_{0} \rightarrow B_{0}} \operatorname{Cut}\left(A_{0} \Rightarrow B_{0}\right)
$$

that leads to the rule

$$
\frac{A_{0}=A_{1} \quad \neg B_{1}, B_{0} \quad C=\left(A_{0} \rightarrow B_{0}\right)}{\left(A_{1} \Rightarrow B_{1}\right), C}
$$

by putting $C=A_{0} \rightarrow B_{0}$. By Lemma [5.4, this rule is admissible, and by Lemma 5.5] so is the rule

$$
\frac{A_{1}, C \quad \neg B_{1}, C}{\neg\left(A_{1} \Rightarrow B_{1}\right), C}
$$

and absorbing the structural rules (in particular contraction on $A \Rightarrow B$ and inversion) leads to the general form

$$
\left(\mathrm{MP}_{g}\right) \frac{A, \neg(A \Rightarrow B), \Gamma \quad \neg B, \neg(A \Rightarrow B), \Gamma}{\neg(A \Rightarrow B), \Gamma}
$$

where we have elided the subscripts. The effect of adding (MP) is similar to that of enriching the modal logic $\mathrm{K}$ with the ( $\mathrm{T})$-axiom. We denote the rule set consisting of all instances of $\mathrm{CK}_{g}$ and $\mathrm{MP}_{g}$ by $\mathrm{CKMP}_{g}$. Our cut elimination theorem then takes the following form:

Proposition 6.5. The rule set $\mathrm{CKMP}_{g}$ is absorbing and equivalent to CKMP.

Proof. It is clear that both $\left(\mathrm{CK}_{g}\right)$ and $\left(\mathrm{MP}_{g}\right)$ absorb the structural rules. For cut, we first consider cuts between two instances of $\left(\mathrm{MP}_{g}\right)$, say

$$
\left(r_{1}\right) \frac{A, \neg(A \Rightarrow B), \Gamma \quad \neg B, \neg(A \Rightarrow B), \Gamma}{\neg(A \Rightarrow B), \Gamma} \quad\left(r_{2}\right) \frac{C, \neg(C \Rightarrow D), \Delta \quad \neg D, \neg(C \Rightarrow D), \Delta}{\neg(C \Rightarrow D), \Delta}
$$

where the cut happens on $F \in \mathcal{F}(\Lambda)$. We distinguish several cases:

Case $F=(A \Rightarrow B)$ and $F \in \Delta$. Then $\Delta=(A \Rightarrow B), \Delta^{\prime}$ for some $\Delta^{\prime} \in \mathrm{S}(\Lambda)$. To eliminate the cut on $C$, we note that the following two derivations

$$
\frac{A, \neg(A \Rightarrow B), \Gamma \quad \neg B, \neg(A \Rightarrow B), \Gamma}{\frac{\neg(A \Rightarrow B), \Gamma \quad(\mathrm{MP}) \quad C, \neg(C \Rightarrow D),(A \Rightarrow B), \Delta^{\prime}}{C, \neg(C \Rightarrow D), \Gamma, \Delta^{\prime}}(\operatorname{cut}(F))}
$$

and

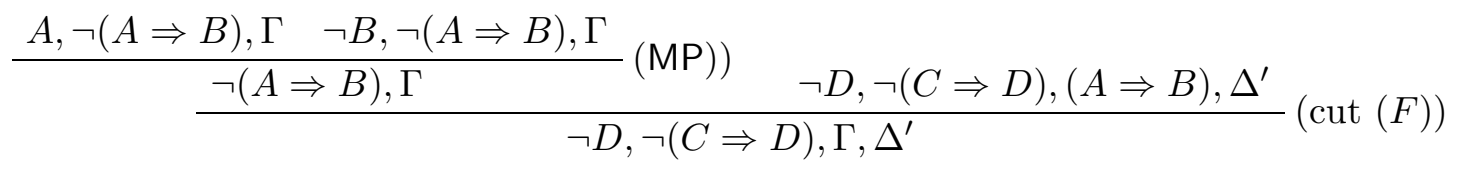

witness that we can use both $C, \neg(C \Rightarrow D), \Gamma, \Delta^{\prime}$ and $\neg D, \neg(C \Rightarrow D), \Gamma, \Delta^{\prime}$ as axioms in $\mathrm{CKMP}_{g}+\operatorname{Cut}\left(F, r_{1}, r_{2}\right)$ as the cuts occur between the premises of $\left(r_{2}\right)$ and conclusions of $\left(r_{1}\right)$. Applying $\left(\mathrm{MP}_{g}\right)$ to these axioms, we obtain that $\mathrm{CKMP}_{g}+\operatorname{Cut}\left(F, r_{1}, r_{2}\right) \vdash \neg(C \Rightarrow D), \Gamma, \Delta^{\prime}$.

Case $F=(C \Rightarrow D)$ and $F \in \Gamma$. This is symmetric to the case above.

Case $F \in \Gamma$ and $\neg F \in \Delta$. Then $\Gamma=\Gamma^{\prime}, F$ and $\Delta=\Delta^{\prime}, \neg F$. We have to show that

$$
\neg(A \Rightarrow B), \neg(C \Rightarrow D), \Gamma^{\prime}, \Delta^{\prime}
$$

is derivable in $\operatorname{Cut}\left(F, r_{1}, r_{2}\right)$. We note that the deduction 


$$
\frac{A, \neg(A \Rightarrow B), F, \Gamma^{\prime} \quad C, \neg(C \Rightarrow D), \neg F, \Delta^{\prime}}{A, \neg(A \Rightarrow B), C, \neg(C \Rightarrow D), \Gamma^{\prime}, \Delta^{\prime}}(\operatorname{Cut}(F))
$$

witnesses that we may use $A, \neg(A \Rightarrow B), C, \neg(C \Rightarrow D), \Gamma^{\prime}, \Delta^{\prime}$ as an axiom in the system $\mathrm{CKMP}_{g}+\operatorname{Cut}\left(F, r_{1}, r_{2}\right)$ as the cut on $F$ has occurred between premises of $r_{1}$ and $r_{2}$. The same deduction, with $C$ replaced by $\neg D$ throughout, witnesses that this is also the case for $A, \neg(A \Rightarrow B), \neg D, \neg(C \Rightarrow D), \Gamma^{\prime}, \Delta^{\prime}$. An application of $\left(\mathrm{MP}_{g}\right)$ now yields $\mathrm{CKMP}_{g}+$ $\operatorname{Cut}\left(F, r_{1}, r_{2}\right) \vdash \neg(C \Rightarrow D), A, \neg(A \Rightarrow B), \Gamma^{\prime}, \Delta^{\prime}$.

By the symmetric argument (just replace $A$ by $\neg B$ ) we obtain that also $\mathrm{CKMP}_{g}+$ Cut $\left(F, r_{1}, r_{2}\right) \vdash \neg(C \Rightarrow D), \neg B, \neg(A \Rightarrow B), \Gamma^{\prime}, \Delta^{\prime}$ and an application of $\left(\mathrm{MP}_{g}\right)$ now yields $\mathrm{CKMP}_{g}+\operatorname{Cut}\left(F, r_{1}, r_{2}\right) \vdash \neg(C \Rightarrow D), \neg(A \Rightarrow B), \Gamma^{\prime}, \Delta^{\prime}$ as required.

What is left is to consider cuts, say on $F \in \mathcal{F}(\Lambda)$, between the conclusions of the rules

$$
\begin{aligned}
& \left(r_{1}\right) \frac{A_{0}=\cdots=A_{n} \quad \neg B_{1}, \ldots, \neg B_{n}, B_{0}}{\neg\left(A_{1} \Rightarrow B_{1}\right), \ldots, \neg\left(A_{n} \Rightarrow B_{n}\right),\left(A_{0} \Rightarrow B_{0}\right), \Gamma} \\
& \left(r_{2}\right) \frac{C, \neg(C \Rightarrow D), \Delta \quad \neg D, \neg(C \Rightarrow D), \Delta}{\neg(C \Rightarrow D), \Delta}
\end{aligned}
$$

As before, we need to discuss several cases.

Case $F \in \Gamma$ or $\neg F \in \Gamma$. Trivial, as the conclusion of the cut can be derived using a different weakening context $\Gamma$.

Case $F=\left(A_{i} \Rightarrow B_{i}\right)$ for some $1 \leq i \leq n$. We assume without loss of generality that $i=1$ and have that $F=\left(A_{1} \Rightarrow B_{1}\right) \in \Delta$ so that $\Delta=\Delta^{\prime}, F$. To replace the cut on $F$, we consider the deduction

$$
\frac{\frac{A_{0}=\cdots=A_{n} \quad \neg B_{1}, \ldots, \neg B_{n}, B_{0}}{\neg\left(A_{1} \Rightarrow B_{1}\right), \ldots, \neg\left(A_{n} \Rightarrow B_{n}\right),\left(A_{0} \Rightarrow B_{0}\right), \Gamma}(\mathrm{CK}) \quad C, \neg(C \Rightarrow D),\left(A_{1} \Rightarrow B_{1}\right), \Delta^{\prime}}{\neg\left(A_{2} \Rightarrow B_{2}\right), \ldots, \neg\left(A_{n} \Rightarrow B_{n}\right),\left(A_{0} \Rightarrow B_{0}\right), C, \neg(C \Rightarrow D), \Gamma, \Delta^{\prime}}(\operatorname{cut}(F))
$$

which witnesses that we may use

$$
\Sigma_{1}=\neg\left(A_{2} \Rightarrow B_{2}\right), \ldots, \neg\left(A_{n} \Rightarrow B_{n}\right),\left(A_{0} \Rightarrow B_{0}\right), C, \neg(C \Rightarrow D), \Gamma, \Delta^{\prime}
$$

as an axiom in $\mathrm{GCKMP}_{g}+\operatorname{Cut}\left(F, r_{1}, r_{2}\right)$. The above deduction, with $C$ replaced by $\neg D$ throughout, witnesses that the same is true for

$$
\Sigma_{2}=\neg\left(A_{2} \Rightarrow B_{2}\right), \ldots, \neg\left(A_{n} \Rightarrow B_{n}\right),\left(A_{0} \Rightarrow B_{0}\right), \neg D, \neg(C \Rightarrow D), \Gamma, \Delta^{\prime}
$$

and applying $\left(\mathrm{MP}_{g}\right)$ with premises $\Sigma_{1}$ and $\Sigma_{2}$ yields $\operatorname{GCKMP}_{g}+\operatorname{Cut}\left(F, r_{1}, r_{2}\right) \vdash \neg\left(A_{2} \Rightarrow\right.$ $\left.B_{2}\right), \ldots, \neg\left(A_{n} \Rightarrow B_{n}\right),\left(A_{0} \Rightarrow B_{0}\right), \neg(C \Rightarrow D), \Gamma, \Delta^{\prime}$ as required.

Case $F=\left(A_{0} \Rightarrow B_{0}\right)=(C \Rightarrow D)$. We have to give a derivation of $\neg\left(A_{1} \Rightarrow\right.$ $\left.B_{1}\right), \ldots, \neg\left(A_{n} \Rightarrow B_{n}\right), \Gamma, \Delta$ in $\operatorname{Cut}\left(F, r_{1}, r_{2}\right)$. The deduction

$$
\frac{\frac{A_{0}=\cdots=A_{n} \quad \neg B_{1}, \ldots, \neg B_{n}, B_{0}}{\neg\left(A_{1} \Rightarrow B_{1}\right), \ldots, \neg\left(A_{n} \Rightarrow B_{n}\right),\left(A_{0} \Rightarrow B_{0}\right), \Gamma}(\mathrm{CK}) \quad \neg B_{0}, \neg\left(A_{0} \Rightarrow B_{0}\right), \Delta}{\neg\left(A_{1} \Rightarrow B_{1}\right), \ldots, \neg\left(A_{n} \Rightarrow B_{n}\right), \neg B_{0}, \Gamma, \Delta}(\operatorname{Cut}(F))
$$

witnesses that we may use

$$
\Sigma_{1}=\neg\left(A_{1} \Rightarrow B_{1}\right), \ldots, \neg\left(A_{n} \Rightarrow B_{n}\right), \neg B_{0}, \Gamma, \Delta
$$


as an axiom in $\mathrm{GCKMP}_{g}+\operatorname{Cut}\left(F, r_{1}, r_{2}\right)$ as the cut on $F$ occurs between a conclusion of $\left(r_{1}\right)$ and a premise of $\left(r_{2}\right)$. The same derivation, with $\neg B_{0}$ replaced by $A_{0}$ shows that the same is true for

$$
\Sigma_{2}=\neg\left(A_{1} \Rightarrow B_{1}\right), \ldots, \neg\left(A_{n} \Rightarrow B_{n}\right), A_{0}, \Gamma, \Delta .
$$

We therefore have the two derivations

$$
\frac{\Sigma_{1} \quad \neg B_{1}, \ldots, \neg B_{n}, B_{0}}{\neg\left(A_{1} \Rightarrow B_{1}\right), \ldots, \neg\left(A_{n} \Rightarrow B_{n}\right), \neg B_{1}, \ldots, \neg B_{n}, \Gamma, \Delta}\left(\operatorname{Cut}\left(B_{0}\right)\right)
$$

and

$$
\frac{\Sigma_{2} \quad \frac{\neg A_{0}, A_{1}}{\neg A_{0}, A_{1}, B_{2}, \ldots, B_{n}}(\mathrm{w})}{\neg\left(A_{1} \Rightarrow B_{1}\right), \ldots, \neg\left(A_{n} \Rightarrow B_{n}\right), A_{1}, B_{2}, \ldots, B_{n}, \Gamma, \Delta}\left(\operatorname{Cut}\left(A_{0}\right)\right)
$$

in $\mathrm{GCKMP}_{g}+\mathrm{Cut}\left(F, r_{1}, r_{2}\right)$. Applying $\left(\mathrm{MP}_{g}\right)$ to the conclusions of both yields that $\mathrm{GCKMP}_{g}+$ Cut $\left(F, r_{1}, r_{2}\right) \vdash \Sigma_{3}$ where

$$
\Sigma_{3}=\neg\left(A_{1}, \Rightarrow B_{1}\right), \ldots, \neg\left(A_{n} \Rightarrow B_{n}\right), \neg B_{2}, \ldots, \neg B_{n}, \Gamma, \Delta
$$

as $\mathrm{GCKMP}_{g}+\operatorname{Cut}\left(F, r_{1}, r_{2}\right)$ contains the contraction rule. We now iterate the same scheme, where we use weakening on a successively smaller subset of $B_{2}, \ldots, B_{n}$. First, we note that

$$
\frac{\Sigma_{2} \quad \frac{\neg A_{0}, A_{2}}{\neg A_{0}, A_{2}, \neg B_{3}, \ldots, \neg B_{n}}(\mathrm{w})}{\neg\left(A_{1} \Rightarrow B_{1}\right), \ldots, \neg\left(A_{n} \Rightarrow B_{n}\right), A_{2}, \neg B_{3}, \ldots, \neg B_{n}, \Gamma, \Delta}\left(\operatorname{Cut}\left(A_{0}\right)\right)
$$

is a derivation in $\mathrm{GCKMP}_{g}+\operatorname{Cut}\left(F, r_{1}, r_{2}\right)$ and applying $\left(\mathrm{MP}_{g}\right)$ to $\Sigma_{3}$ and its conclusion yields $\mathrm{GCKMP}_{g}+\operatorname{Cut}\left(F, r_{1}, r_{2}\right) \vdash \Sigma_{4}$ where

$$
\Sigma_{4}=\neg\left(A_{1} \Rightarrow B_{1}\right), \ldots, \neg\left(A_{n} \Rightarrow B_{n}\right), \neg B_{3}, \ldots, \neg B_{n}
$$

Iterating this scheme, we finally obtain

$$
\operatorname{GCKMP}_{g}+\operatorname{Cut}\left(F, r_{1}, r_{2}\right) \vdash \neg\left(A_{1} \Rightarrow B_{1}\right), \ldots, \neg\left(A_{n} \Rightarrow B_{n}\right), \Gamma, \Delta .
$$

Note that weakening and cuts on formulas of size $<\operatorname{size}(F)$ is admissible in $\mathrm{GCKMP}_{g}+$ Cut $\left(F, r_{1}, r_{2}\right)$.

Case $F=\left(A_{0} \Rightarrow B_{0}\right)$ and $\neg F \in \Delta$. We have that $\Delta=\neg\left(A_{0} \Rightarrow B_{0}\right), \Delta^{\prime}$ and the deduction

$$
\frac{\frac{A_{0}=\cdots=A_{n} \quad \neg B_{1}, \ldots, \neg B_{n}, B_{0}}{\neg\left(A_{1} \Rightarrow B_{1}\right), \ldots, \neg\left(A_{n} \Rightarrow B_{n}\right),\left(A_{0} \Rightarrow B_{0}\right), \Gamma}(\mathrm{CK}) \quad C, \neg(C \Rightarrow D), \neg\left(A_{0} \Rightarrow B_{0}\right), \Delta^{\prime}}{\neg\left(A_{1} \Rightarrow B_{1}\right), \ldots, \neg\left(A_{n} \Rightarrow B_{n}\right), C, \neg(C \Rightarrow D), \Gamma, \Delta^{\prime}}(\operatorname{Cut}(F))
$$

witnesses that we may use

$$
\Sigma_{2}=\neg\left(A_{1} \Rightarrow B_{1}\right), \ldots, \neg\left(A_{n} \Rightarrow B_{n}\right), C, \neg(C \Rightarrow D), \Gamma, \Delta^{\prime}
$$

as an axiom in $\mathrm{GCKMP}_{g}+\operatorname{Cut}\left(F, r_{1}, r_{2}\right)$. The same derivation, with $C$ replaced by $\neg D$, shows that the same is true for

$$
\Sigma_{2}=\neg\left(A_{1} \Rightarrow B_{1}\right), \ldots, \neg\left(A_{n} \Rightarrow B_{n}\right), \neg D, \neg(C \Rightarrow D), \Gamma, \Delta^{\prime}
$$

and applying $\left(\mathrm{MP}_{g}\right)$ with premises $\Sigma_{1}$ and $\Sigma_{2}$ yields the claim $\operatorname{GCKMP}_{g}+\operatorname{Cut}\left(F, r_{1}, r_{2}\right) \vdash$ $\neg\left(A_{1} \Rightarrow B_{1}\right), \ldots, \neg\left(A_{n} \Rightarrow B_{n}\right), \Gamma, \Delta^{\prime}$. This finishes our analysis of cuts that may arise between conclusions of the $\left(\mathrm{CK}_{g}\right)$ and the $\left(\mathrm{MP}_{g}\right)$-rule, and hence the proof. 
As an example, we give a derivation of (MP) in the system $\mathrm{CKMP}_{g}$ (in fact, a single application of $\left(\mathrm{MP}_{g}\right)$ suffices $)$.

Example 6.6. If expressed solely in terms of $\wedge$ and $\neg$, conditional modus ponens takes the form $\neg((A \Rightarrow B) \wedge A \wedge \neg B)$. The following derivation establishes that (MP) is derivable in the above form

$$
\begin{aligned}
A, \neg(A \Rightarrow B), \neg A, B \quad \neg B, \neg(A \Rightarrow B), \neg A, B \\
\frac{}{\frac{\neg(A \Rightarrow B), \neg A, B}{}}\left(\mathrm{MP}_{g}\right) \\
\frac{\neg(A \Rightarrow B), \neg A, \neg \neg B}{\neg((A \Rightarrow B), \neg(A \wedge \neg B)}(\neg \wedge) \\
\neg(\neg) \wedge A \wedge \neg B)
\end{aligned}
$$

so that (MP) is derivable in $\mathrm{CKMP}_{g}$.

We now consider the logic that arises by adding both conditional modus ponens $(A \Rightarrow$ $B) \rightarrow(A \rightarrow B)$ and the identity axiom $A \Rightarrow A$ to the logic CK. In line with our naming conventions, this logic is called CKMPID. To obtain a cut-free axiomatisation of this logic, we consider the rule set $\mathrm{CKMPID}_{g}$ containing all instances of $\mathrm{CKID}_{g}$ and $\mathrm{MP}_{g}$. A close inspection of the proof of Proposition 6.5 gives that $\mathrm{CKMPID}_{g}$ is absorbing, and therefore cut-free complete.

Proposition 6.7. The rule set $\mathrm{CKMPID}_{g}$ is absorbing and equivalent to CKMPID.

Proof. We follow the same strategy (and consider the same cases) as in the proof of Proposition 6.5 where we note that the conclusions of $\mathrm{CK}_{g}$ and $\mathrm{CKID}_{g}$ are identical, the only difference being that in premise displayed on the far right in $\mathrm{CK}_{g}$ and $\mathrm{CKID}_{g}$ there is one additional (negative) literal in where $\mathrm{CKID}_{g}$. The proof of Proposition 6.5 can now be repeated literally by adding this extra literal to all instances of $\mathrm{CK}_{g}$, thus turning every instance of $\mathrm{CK}_{g}$ in the proof of Proposition 6.5 into an instance of $\mathrm{CKID}_{g}$.

6.2. Cut Elimination for Extensions of CKCEM. To construct an absorbing rule set for conditional logic plus the axiom

$$
(\mathrm{CEM})(A \Rightarrow B) \vee(A \Rightarrow \neg B)
$$

we start from the admissible rule set for CK and close under cuts that arise with (CEM). Repeated applications of Lemma 5.4 and Lemma 5.5 lead to the rule set

$$
\left(\operatorname{CKCEM}_{g}\right) \frac{A_{0}=\cdots=A_{n} \quad B_{0}, \ldots, B_{j}, \neg B_{j+1}, \neg B_{n}}{\left(A_{0} \Rightarrow B_{0}\right), \ldots,\left(A_{j} \Rightarrow B_{j}\right), \neg\left(A_{j+1} \Rightarrow B_{j+1}\right), \ldots, \neg\left(A_{n} \Rightarrow B_{n}\right), \Gamma}
$$

for $0 \leq j \leq n$.

Proposition 6.8. The rule set $\mathrm{CKCEM}_{g}$ is absorbing and equivalent to CKCEM.

Proof. Again, it suffices to check that the rule set $\mathrm{CKCEM}_{g}$ is absorbing, where the absorption of structural rules is clear. It therefore suffices to treat instances of cuts between 
conclusions of rules of CKCEM. Owing to the form of the $\mathrm{CKCEM}_{g}$-rule, our argument is very similar to that used for $\mathrm{CK}_{g}$. We consider the following two instances

$$
\begin{gathered}
\left(r_{1}\right) \frac{A_{0}=\cdots=A_{n} \quad B_{0}, \ldots, B_{i}, \neg B_{i+1}, \ldots, \neg B_{n}}{\left(A_{0} \Rightarrow B_{0}\right), \ldots,\left(A_{i} \Rightarrow B_{i}\right), \neg\left(A_{i+1} \Rightarrow B_{i+1}\right), \ldots, \neg\left(A_{n} \Rightarrow B_{n}\right), \Gamma} \\
\left(r_{2}\right) \frac{C_{0}=\cdots=C_{m} \quad D_{0}, \ldots, D_{j}, \neg D_{j+1}, \ldots, \neg D_{m}}{\left(C_{0} \Rightarrow D_{0}\right), \ldots,\left(C_{j} \Rightarrow D_{j}\right), \neg\left(C_{j+1} \Rightarrow D_{j+1}\right), \ldots, \neg\left(C_{m} \Rightarrow D_{m}\right), \Delta}
\end{gathered}
$$

and assume that the conclusions permit an instance of cut on $F \in \mathcal{F}(\Lambda)$. As usual, we distinguish several cases, where the cases $F \in \Gamma, F \in \Delta, \neg F \in \Gamma$ and $\neg F \in \Delta$ are trivial.

Case $F=\left(A_{k} \Rightarrow B_{k}\right)=\left(C_{l} \Rightarrow D_{l}\right)$ for $k>i$ and $0 \leq l \leq j$. Without loss of generality we assume that $k=n$ and $l=0$ and get $A_{n}=C_{0}$ and $B_{n}=D_{0}$. Denote the sequent that arises from applying cut on $F$ to the conclusions of $r_{1}$ and $r_{2}$ by $\Sigma_{0}$ and notice that, using cuts on $A_{n} \equiv C_{0}$, we have that

$$
\Sigma=A_{0}=A_{1}=\cdots=A_{n-1}=C_{1}=\cdots=C_{m}
$$

is derivable in $\operatorname{GCKCEM}_{g}+\operatorname{Cut}\left(F, r_{1}, r_{2}\right)$. This feeds into the derivation

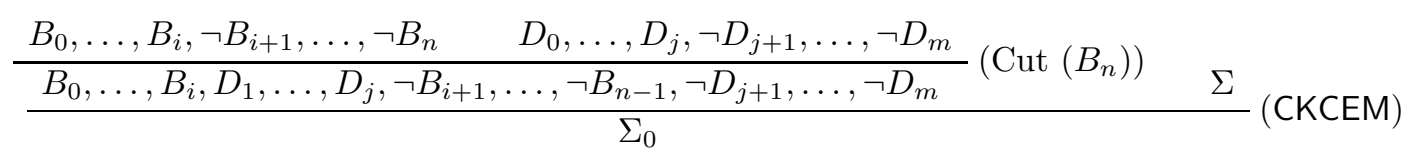

which establishes that $\operatorname{Cut}\left(F, r_{1}, r_{2}\right) \vdash \Sigma_{0}$ as desired.

The case $F=\left(A_{k} \Rightarrow B_{k}\right) \equiv\left(B_{l} \Rightarrow D_{l}\right)$ for $k>i$ and $1 \leq l \leq j$ is symmetric, which finishes the proof.

As a consequence, cut elimination holds in $\mathrm{CKCEM}_{g}$. We show of the (CEM) can be derived before moving on to the next calculus.

Example 6.9. If we spell out the abbreviatios of $\vee$ in terms of $\neg$ and $\wedge$, the axiom of conditional excluded middle takes the form $\neg(\neg(A \Rightarrow B) \wedge \neg(A \Rightarrow \neg B))$. The following derivation in $\mathrm{CKCEM}_{g}$ shows that this form of the axiom is derivable.

$$
\begin{gathered}
\frac{A=A \quad B, \neg B}{(A \Rightarrow B),(A \Rightarrow \neg B)}\left(\text { CKCEM }_{g}\right) \\
\frac{\neg \neg(A \Rightarrow B),(A \Rightarrow \neg B)}{\neg \neg)} \\
\frac{\neg \neg(A \Rightarrow B), \neg \neg(A \Rightarrow \neg B)}{\neg(\neg(A \Rightarrow B) \wedge \neg(C \Rightarrow \neg B)}(\neg \wedge)
\end{gathered}
$$

In this derivation, we have chosen $j=n=1$ and when applying $\left(\mathrm{CKCEM}_{g}\right)$ and choosing $j=0$ yields an instance of $\left(\mathrm{CK}_{g}\right)$.

We now consider the extension of $\mathrm{CK}$ with both conditional excluded middle $(A \Rightarrow B) \vee(A \Rightarrow$ $\neg B)$ and the identity axiom $(A \Rightarrow A)$ and denote the ensuing logic by CKCEMID. As in the construction of the rule set $\mathrm{CKID}_{g}$, we construct a rule set by applying Lemma 5.4 and 5.5 by considering cuts between an instance of $\left(\mathrm{CKCEM}_{g}\right)$ (left) and a rule arising from a cut between $\left(\mathrm{CK}_{g}\right)$ and the identity axiom (right)

$$
\left(\mathrm{CKCEM}_{g}\right) \frac{A_{0}=\cdots=A_{n} \quad B_{0}, \ldots, B_{j}, \neg B_{j+1}, \neg B_{n}}{\left(A_{0} \Rightarrow B_{0}\right), \ldots,\left(A_{j} \Rightarrow B_{j}\right), \neg\left(A_{j+1} \Rightarrow B_{j+1}\right), \ldots, \neg\left(A_{n} \Rightarrow B_{n}\right), \Gamma} \quad \frac{\neg C, D}{C \Rightarrow D}
$$


(where $1 \leq j \leq n$ ) leads to the (admissible) rule schema

$$
\left(\text { CKCEMID }_{g}\right) \frac{A_{0}=\cdots=A_{n} \quad \neg A_{0}, B_{0}, \ldots, B_{j}, \neg B_{j+1}, \neg B_{n}}{\left(A_{0} \Rightarrow B_{0}\right), \ldots,\left(A_{j} \Rightarrow B_{j}\right), \neg\left(A_{j+1} \Rightarrow B_{j+1}\right), \ldots, \neg\left(A_{n} \Rightarrow B_{n}\right), \Gamma}
$$

that provides a cut-free axiomatisation of CKCEMID, as we now show.

Proposition 6.10. The rule set $\mathrm{CKCEMID}_{g}$ is equivalent to CKCEMID and absorbing.

Proof. Just as in the proof of Proposition 6.8 we consider cuts between two instances of $\left(\mathrm{CKCEMID}_{g}\right)$ and note that the premises of $\left(\mathrm{CKCEM}_{g}\right)$ and $\left(\mathrm{CKCEMID}_{g}\right)$ only differ by a negative literal that is added to the premise on the far right in $\left(\mathrm{CKCEMID}_{g}\right)$. We consider precisely the same cases as in the proof of Proposition 6.8. Using the same notation, we note that the equality $A_{0}=\cdots=A_{n}$ in particular gives $\neg A_{n}, A_{0}$ as a premise, and we use an additional cut on $A_{0}$, followed by an instance of contraction, immediately prior to the application of (CKCEM) (that we replace by an instance of $\left(\mathrm{CKCEMID}_{g}\right)$ ) to show absorption of cut.

We now consider extending CK with both conditional modus ponens and conditional excluded middle, but have to take care of the cuts arising between $\mathrm{MP}_{g}$ and $\mathrm{CKCEM}_{g}$, which leads to the new rule

$$
\left(\text { MPCEM }_{g}\right) \frac{A,(A \Rightarrow B), \Gamma \quad B,(A \Rightarrow B), \Gamma}{(A \Rightarrow B), \Gamma}
$$

that was obtained in the same way as $\left(\mathrm{MP}_{g}\right)$. If we denote the extension of $\mathrm{CKCEM}_{g}$ with $\mathrm{MP}_{g}$ and $\mathrm{MPCEM}_{g}$ by CKCEMMP ${ }_{g}$, we obtain:

Proposition 6.11. CKCEMMP $_{g}$ is absorbing and equivalent to CKCEMMP.

Proof. It is clear that the rule set CKCEMMP $g$ absorbs the structural rules and it is easy to see that it is equivalent to CKCEMMP. We have to show that it absorbs cut.

Cuts between the conclusions of two instances of $\mathrm{MP}_{g}$ have already been treated in the proof of Theorem 6.5, and the proof translates verbatim to cuts between instances of MPCEM $_{g}$. We consider cuts between two instances

$$
\left(r_{1}\right) \frac{A,(A \Rightarrow B), \Gamma \quad B,(A \Rightarrow B), \Gamma}{(A \Rightarrow B), \Gamma} \quad\left(r_{2}\right) \frac{C, \neg(C \Rightarrow D), \Delta \quad \neg D, \neg(C \Rightarrow D), \Delta}{\neg(C \Rightarrow D), \Delta}
$$

where the cut is performed on $F \in \mathcal{F}(\Lambda)$, say. The cases where either $F \in \Gamma$ and $\neg F$ in $\Delta$ or $F \in \Delta$ and $\neg F \in \Gamma$ are straightforward.

Case $F=(A \Rightarrow B)=(C \Rightarrow D)$. The derivation

$$
\frac{A,(A \Rightarrow B), \Gamma \quad B,(A \Rightarrow B), \Gamma}{\frac{(A \Rightarrow B), \Gamma \quad}{(\text { MPCEM })} \quad \neg B, \neg(A \Rightarrow B), \Delta}(\operatorname{Cut}(A \Rightarrow B, \Gamma, \Delta))
$$

witnesses that we may use $\Sigma_{1}=\neg B, \Gamma, \Delta$ as an axiom in $\operatorname{GCKCEMMP}+\operatorname{Cut}\left(F, r_{1}, r_{2}\right)$. Similarly, the derivation

$$
\frac{A, \neg(A \Rightarrow B), \Delta \quad \neg B, \neg(A \Rightarrow B), \Delta}{\frac{\neg(A \Rightarrow B), \Delta}{B, \Gamma, \Delta} \quad B,(A \Rightarrow B), \Gamma}(\operatorname{Cut}(A \Rightarrow B))
$$


shows that the same is true for $\Sigma_{2}=B, \Gamma, \Delta$ : note that in both cases, the cut was performed between an axiom and a conclusion of both rules. As size $(B)<\operatorname{size}(A \Rightarrow B)$ we may now use cut on $B$ to establish that GCKCEMMP $g+\operatorname{Cut}\left(F, r_{1}, r_{2}\right) \vdash \Gamma, \Delta$.

Case $F=(A \Rightarrow B)$ and $\neg F \in \Delta$. We have that $\Delta=\neg(A \Rightarrow B), \Delta^{\prime}$. The derivation

$$
\frac{A,(A \Rightarrow B), \Gamma \quad B,(A \Rightarrow B), \Gamma}{\frac{(A \Rightarrow B), \Gamma \quad(\text { MPCEM }) \quad C, \neg(C \Rightarrow D), \neg(A \Rightarrow B), \Delta^{\prime}}{C, \neg(C \Rightarrow D), \Gamma, \Delta^{\prime}}}(\operatorname{Cut}(F))
$$

witnesses that we may use

$$
\Sigma_{1}=C, \neg(C \Rightarrow D), \Gamma, \Delta^{\prime}
$$

as an axiom in GCKCEMMP $+\operatorname{Cut}\left(F, r_{1}, r_{2}\right)$. The same derivation, with $C$ replaced by $\neg D$ shows that the same is true for

$$
\Sigma_{2}=\neg D, \neg(C \Rightarrow D), \Gamma, \Delta^{\prime}
$$

and an application of $\mathrm{MP}_{g}$ yields derivability of $\neg(C \Rightarrow D), \Gamma, \Delta^{\prime}$.

Case $F=(C \Rightarrow D)$ and $\neg F \in \Gamma$. Analogous by interchaning the role of $\mathrm{MP}_{g}$ and $\mathrm{MPCEM}_{g}$.

This leaves to consider cuts between two instances of $\mathrm{CKCEM}_{g}$ and $\mathrm{MP}_{g}$ and between $\mathrm{CKCEM}_{g}$ and MPCEM $_{g}$. We first consider the rules

$$
\begin{aligned}
& \left(r_{1}\right) \frac{A,(A \Rightarrow B), \Gamma \quad B,(A \Rightarrow B), \Gamma}{(A \Rightarrow B), \Gamma} \\
& \left(r_{2}\right) \frac{A_{0}=\cdots=A_{n} \quad B_{0}, \ldots, B_{j}, \neg B_{j+1}, \ldots, \neg B_{n}}{A_{0} \Rightarrow B_{0}, \ldots,\left(A_{j} \Rightarrow B_{j}\right), \neg\left(A_{j+1} \Rightarrow B_{j+1}\right), \ldots, \neg\left(A_{n} \Rightarrow B_{n}\right), \Delta}
\end{aligned}
$$

In this setting, all cases except the case $F=(A \Rightarrow B)=\left(A_{i} \Rightarrow B_{i}\right)$ with $i>j$ are entirely analogous to those considered in the proof of Theorem 6.5 where applications of $\mathrm{CK}_{g}$ need to be replaced by applications of $\mathrm{CKCEM}_{g}$. In case $F=(A \Rightarrow B)=\left(A_{i} \Rightarrow B_{i}\right)$ with $i>j$ we assume without loss of generality that $i=n$ and argue, as in the proof of Theorem 6.5, that

$$
\Sigma_{1}=\left(A_{0} \Rightarrow B_{0}\right), \ldots,\left(A_{j} \Rightarrow B_{j}\right), \neg\left(A_{j+1} \Rightarrow B_{j+1}\right), \ldots, \neg\left(A_{n-1} \Rightarrow B_{n-1}\right), B_{n}, \Gamma, \Delta
$$

and

$$
\Sigma_{2}=\left(A_{0} \Rightarrow B_{0}\right), \ldots,\left(A_{j} \Rightarrow B_{j}\right), \neg\left(A_{j+1} \Rightarrow B_{j+1}\right), \ldots, \neg\left(A_{n-1} \Rightarrow B_{n-1}\right), A_{n}, \Gamma, \Delta
$$

both are axioms of GCKCEMMP $g$ + Cut $\left(F, r_{1}, r_{2}\right)$, leading to deductions ending in, repectively, $\Sigma_{1}, B_{0}, \ldots, B_{j}, \neg B_{j+1}, \ldots, \neg B_{n-1}$ and $\Sigma_{2}, \neg A_{0}, B_{1}, \ldots, B_{j}, \neg B_{j+1}, \ldots \neg B_{n-1}$. An application of $\mathrm{MP}_{g}$ now yields derivability of $\left(A_{0} \Rightarrow B_{0}\right), \ldots,\left(A_{j} \Rightarrow B_{j}\right), \neg\left(A_{j+1} \Rightarrow\right.$ $\left.B_{j+1}\right), \ldots, \neg\left(A_{n-1} \Rightarrow B_{n-1}\right), B_{1}, \ldots, B_{j}, \neg B_{j+1}, \ldots, \neg B_{n-1}$. Iterating the same schema, where MPCEM $_{g}$ is used instead of $\mathrm{MP}_{g}$ to eliminate occurrences of $\neg B_{i}$ for $i>j$ finally yields that $\left(A_{0} \Rightarrow B_{0}\right), \ldots,\left(A_{j} \Rightarrow B_{j}\right), \neg\left(A_{j+1} \Rightarrow B_{j+1}\right), \ldots, \neg\left(A_{n-1} \Rightarrow B_{n-1}\right), \Gamma, \Delta$ is derivable in $\mathrm{GCKCEMMP}_{g}+\operatorname{Cut}\left(F, r_{1}, r_{2}\right)$.

To see that cuts between conclusions of $\mathrm{CKCEM}_{g}$ and $\mathrm{MPCEM}_{g}$ can be eliminated, one uses the same reasoning as above, with $\mathrm{MPCEM}_{g}$ and $\mathrm{MP}_{g}$ interchanged. 
We note that the latter theorem was left as an open problem for the sequent system presented in [13. To complete the treatment of conditional logics, we now turn to the system CKCEMMPID that arises by extending CK with the axioms correspoinding to conditional excluded middle, conditional modus ponens and identity. It follows by construction that the rule set CKCEMMPID ${ }_{g}$, that we take as containing all instances of CKCEMID,$\left(\mathrm{MP}_{g}\right)$ and $\left(\mathrm{MPCEM}_{g}\right)$ induces a calculus that is equivalent to CKCEMMPID and we just have to establish absorption.

Proposition 6.12. The rule set $\mathrm{CKCEMMPID}_{g}$ is equivalent to $\mathrm{CKCEMMPID}_{g}$ and absorbing.

Proof. It is clear that CKCEMMPID $g$ absorbs the structural rules (this was established before for each rule schema). To see that cut is absorbed, we proceed as in the proof of Proposition 6.11 where we replace every occurrence of $\left(\mathrm{CKCEM}_{g}\right)$ by the corresponding instance of $\left(\mathrm{CKCEMID}_{g}\right)$. The additional literal in the rightmost premise of $\left(\mathrm{CKCEMID}_{g}\right)$ is treated in the same way as in the proof of Proposition 6.3.

In summary, we obtain the following results about extensions of the conditional logic CK.

Theorem 6.13. Suppose that $\mathrm{L}$ is a combination of ID, MP, CEM. Then $\mathrm{GL}_{g} \vdash A$ whenever $\mathrm{HL} \vdash A$ for all $A \in \mathcal{F}(\Lambda)$. Moreover, cut elimination holds in $\mathrm{GL}$.

The theorem follows, in each of the cases, from Theorem 4.6 and Theorem 5.3 together with the fact that the rule set $L$ and $L_{g}$ are equivalent and the latter is absorbing.

\section{Complexity of Proof Search}

It is comparatively straightforward to extract complexity bounds for provability of the logics considered above by analysing the complexity of proof search under suitable strategies in the cut-free sequent systems obtained. Clearly, in those cases where all modal rules peel off exactly one layer of modal operators, the depth of proofs is polynomial in the nesting depth of modal operators in the target formula, and therefore, proof search is in PSPACE under mild assumptions on the branching width of proofs [18, 14]. Besides reproving Ladner's classical result for $K$ [12], we thus have

Theorem 7.1. Provability in $\mathrm{CK}$ and CKID is in PSPACE.

This reproves known complexity bounds originally shown in [13] (alternative short proofs using coalgebraic semantics are given in [17]). For CKCEM, the bound can be improved to coNP using dynamic programming in the same style as in 20]. This concept has to be handled carefully when dealing with coNP bounds, however, as in nondeterministic programs we cannot actually pretend that during the execution of stage $n$ we have the results of the stages up to $n-1$ stored in memory - otherwise, we could, e.g., just negate these results and arrive at proving $\mathrm{NP}=$ coNP. Rather, dynamic programming should be regarded as a metaphor for merging identical computations on a non-deterministic machine; in particular, we need to take care to use results of previous stages only positively (as done in 20$]$ ).

The point in our decision procedure where these considerations become relevant is that we will wish to apply rule $\left(\mathrm{CKCEM}_{g}\right)$ deterministically to subsequents that are as large as possible; i.e. we are interested in collecting maximal sets of conditional literals with provably 
equivalent antecedents, where the latter equivalences are supposed to have been computed in previous stages. Here, the maximality condition carries the danger of negative use of previous results. The solution to this problem lies in the following key lemma.

Lemma 7.2. Let $\Gamma=\left(A_{0} \Rightarrow B_{0}\right), \ldots,\left(A_{j} \Rightarrow B_{j}\right), A_{j+1} \Rightarrow B_{j+1}, \ldots, A_{n} \Rightarrow B_{n}$ be a sequent consisting of conditional literals. Then $\Gamma$ is provable in $\mathrm{CKCEM}_{g}$ iff for every decomposition of $\{0, \ldots, n\}$ into disjoint sets $I_{0}, \ldots, I_{k}(k \geq 0)$, one of the following conditions holds.

(1) There exists $l$ such that $\left\{B_{i} \mid i \in I_{l}, 0 \leq i \leq j\right\} \cup\left\{\neg B_{i} \mid i \in I_{l}, j+1 \leq i \leq n\right\}$ is provable.

(2) There exist $l \neq r$ and $i \in I_{l}, p \in I_{r}$ such that $A_{i}=A_{p}$ is provable.

Proof. Only if: Since $\Gamma$ consists of conditional literals, any proof of $\Gamma$ must end in an application of rule $\mathrm{CKCEM}_{g}$. Thus, there exists $I \subseteq\{0, \ldots, n\}$ such that $A_{i}=A_{p}$ is provable for all $i, p \in I$ and $\left\{B_{i} \mid i \in I, 0 \leq i \leq j\right\} \cup\left\{\neg B_{i} \mid i \in I, j+1 \leq i \leq n\right\}$ is provable. Now let $I_{o}, \ldots, I_{k}$ be as in the statement. Then we have the following two cases:

(1) There exists $l$ such that $I \subseteq I_{l}$. In this case, the first alternative of the claim holds.

(2) We have $i, p \in I$ and $r \neq l$ such that $i \in I_{r}, p \in I_{l}$. In this case, the second alternative of the claim holds.

If: Define an equivalence relation on $\{0, \ldots, n\}$ by taking $i$ and $p$ to be equivalent if $A_{i}=A_{p}$ is provable, and let $I_{1}, \ldots, I_{k}$ be the induced disjoint decomposition of $\{0, \ldots, n\}$ into equivalence classes. By construction, this decomposition does not satisfy the second alternative of the claim, hence it satisfies the first, which implies that $\Gamma$ is provable by applying rule $\mathrm{CKCEM}_{g}$.

This lemma now enables us to prove the announced coNP upper bound:

Theorem 7.3. Provability in CKCEM and in CKIDCEM is in coNP.

Proof. Since some aspects of our algorithm are more easily understood in NP style, we prove that unprovability of a sequent $\Gamma$ can be decided in NP. We use dynamic programming as in [20]: we proceed in stages; at stage $i$, we decide unprovability of all sequents of the form $A, \neg B$ where $A$ and $B$ are subformulas of $\Gamma$ with nesting depth of conditionals at most $i$. We perform such stages up to $i=m-1$, where $m$ is the maximal nesting depth of conditionals in $\Gamma$. In a further, final stage, we then check unprovability of $\Gamma$. As there are at most linearly many stages, it suffices to show that each stage can be performed in NP, and since there are at most quadratically many candidate sequents in each stage, it suffices that unprovability of a single candidate sequent can be checked in NP at each stage.

To this end, observe that proofs may generally be normalised to proceed as follows: first apply the propositional rules as long as possible, thus decomposing target sequents into sequents over conditional literals, i.e. literals of the form $A \Rightarrow B$ or $\neg(A \Rightarrow B)$, in the various branches of the proof, and only apply $\left(\mathrm{CKCEM}_{g}\right)$ when no more propositional rules are applicable (since all propositional rules monotonically increase the set of conditional literals when moving from the conclusion to the premises, it is clear that their - backwards application never obstructs a possible application of $\left.\left(\mathrm{CKCEM}_{g}\right)\right)$. The existential branching that arises from the conjunction rule $(A \wedge B, \Delta$ is unprovable if either $A, \Delta$ or $B, \Delta$ is unprovable) is handled non-deterministically. It is clear that one can apply only linearly many propositional rules in any given branch of the computation.

The application of rule $\left(\mathrm{CKCEM}_{g}\right)$ after exhaustion of the propositional rules is handled according to Lemma 7.2 . to check that a sequent of the form $\Delta=A_{0} \Rightarrow B_{0}, \ldots, A_{j} \Rightarrow$ $B_{j}, \neg\left(A_{j+1} \Rightarrow B_{j+1}\right), \ldots, \neg\left(A_{n} \Rightarrow B_{n}\right)$ is unprovable, we guess a disjoint decomposition 
$I_{1}, \ldots, I_{k}$ of $\{0, \ldots, n\}$ and check that it violates Conditions (1) and (2) in the Lemma; the negation of these conditions introduces universal quantifiers over polynomial-sized ranges, which we check deterministically. Here, checking violation of Condition (2) amounts to using unprovability of quadratically many sequents checked in previous stages, which from the perspective of the present stage can be done in polynomial time. Checking violation of Condition (1) is more problematic, as it involves a recursive check of unprovability of sequents $\Delta_{l}=\left\{B_{i} \mid i \in I_{l}, 0 \leq i \leq j\right\} \cup\left\{\neg B_{i} \mid i \in I_{l}, j+1 \leq i \leq n\right\}$ for all $1 \leq l \leq k$; specifically, we have to ensure that this recursion, whose depth is limited by the nesting depth of conditionals in $\Gamma$, does not lead to exponentially long computation paths.

To this end, we note that the breadth of the part of the proof tree that we explore in one computation is given by a function $f(\Delta)$ that obeys a recursive equation of the form

$$
f(\Delta)=\sum_{l=0}^{k} f\left(\Delta_{l}\right)
$$

where

$$
\operatorname{size}(\Delta) \geq \sum_{l=0}^{k} \operatorname{size}\left(\Delta_{l}\right)
$$

because $I_{0}, \ldots, I_{k}$ is a disjoint decomposition of $\{0, \ldots, n\}$. It follows easily that $f(\Delta)$ is at most linear in $\Delta$, and hence the overall size of the part of the proof tree explored in one computation is at most quadratic. This finishes the proof for the case of CKCEM. The proof for CKIDCEM is entirely analogous, noting that although the main premise in rule $\left(\mathrm{CKIDCEM}_{g}\right)$ is by one literal $\neg A_{0}$ larger that in the case of $\left(\mathrm{CKCEM}_{g}\right)$, estimate (7.1) remains true.

More interesting are those cases where some of the modal operators from the conclusion remain in the premise, such as T, K4, CKMP, and CKMPCEM (where the difference between non-iterative logics, i.e. ones whose Hilbert-axiomatisation does not use nested modalities, such as T, CKMP, and CKMPCEM, and iterative logics such as K4 is surprisingly hard to spot in the sequent presentation). For K4, the standard approach is to consider proofs of minimal depth, which therefore never attempt to prove a sequent repeatedly, and analyse the maximal depth that a branch of a proof can have without repeating a sequent. For $\mathrm{T}$, a different strategy is used, where the $(T)$ rule is limited to be applied at most once to every formula of the form $\neg \square A$ in between two applications of $(K)$ [10]. A similar strategy works for the conditional logics CKMP and CKMPCEM, which we explain in some additional detail for CKMP.

We let $\mathrm{CKMP}_{g}^{0}$ and $\mathrm{CKMP}_{g}^{1}$ denote restricted sequent systems, defined as follows.

- In $\mathrm{CKMP}_{g}^{0}$, a formula $\neg(A \Rightarrow B)$ is marked on a branch as soon as the rule $\left(\mathrm{MP}_{g}\right)$ has been applied to it (backwards) and unmarked only at the next application of rule $\left(\mathrm{CK}_{g}\right)$. Rule $\left(\mathrm{MP}_{g}\right)$ applies only to unmarked formulas.

- In $\mathrm{CKMP}_{g}^{1}$, we instead impose a similar restriction where rule $\left(\mathrm{MP}_{g}\right)$ applies to a sequent $\neg(A \Rightarrow B), \Gamma$ only in case $\Gamma$ does not contain a propositional descendant of either $A$ or $\neg B$. Here, a sequent $\Delta$ is called a propositional descendant of a formula $A$ if it can be generated from $A$ by applying propositional sequent rules backwards. Formally, the set $\mathcal{D}$ of propositional descendants of $A$ is the closure of $\{A\}$ under the inversion rules. (E.g. the propositional descendants of $(\neg(A \wedge B) \wedge C)$ are $(\neg(A \wedge B) \wedge C) ; \neg(A \wedge B) ; C$; and $\neg A, \neg B$.) 
Our goal is to show that $\mathrm{CKMP}_{g}, \mathrm{CKMP}_{g}^{0}$, and $\mathrm{CKMP}_{g}^{1}$ prove the same sequents. Here, two inclusions are easy to show:

Lemma 7.4. Every sequent that is provable in $\mathrm{CKMP}_{g}^{1}$ is provable in $\mathrm{CKMP}_{g}^{0}$, and every sequent that is provable in $\mathrm{CKMP}_{g}^{0}$ is provable in $\mathrm{CKMP}_{g}$.

Proof. The second implication is trivial, since $\mathrm{CKMP}_{g}^{0}$ explicitly restricts $\mathrm{CKMP}_{g}$. The first implication follows from the fact that whenever an occurrence of a formula $\neg(A \Rightarrow B)$ is marked in a sequent $\neg(A \Rightarrow B), \Gamma$ in a $\mathrm{CKMP}_{g}^{0}$ proof, then $\Gamma$ must contain a propositional descendant of either $A$ or $\neg B$.

Next, we observe:

Lemma 7.5. The system $\mathrm{CKMP}_{g}^{1}$ admits inversion.

Proof. The inductive proof for $\mathrm{CKMP}_{g}$ can just be copied due to the fact that absorption of inversion by $\mathrm{CKMP}_{g}$ never involves the introduction of additional applications of $\left(\mathrm{MP}_{g}\right)$, and the conclusion of instances of inversion never introduces additional propositional descendants (unlike, e.g., in the case of weakening).

This enables us to prove the missing inclusion:

Lemma 7.6. Every sequent that is provable in $\mathrm{CKMP}_{g}$ is provable in $\mathrm{CKMP}_{g}^{1}$.

Proof. By Lemma 7.5, it suffices to prove that we can replace backwards applications of $\left(\mathrm{MP}_{g}\right)$ to sequents $\neg(A \Rightarrow B), \Gamma$ with $\Gamma$ containing a propositional descendant of either $A$ or $\neg B$, with subproofs using inversion. This is clear: e.g. if $\Gamma$ contains a propositional descendant of $A$, then $\neg(A \rightarrow B), \Gamma$ can be proved from $\neg(A \rightarrow B), A, \Gamma$ alone by repeated application of inversion.

Corollary 7.7. The systems $\mathrm{CKMP}_{g}$ and $\mathrm{CKMP}_{g}^{0}$ prove the same sequents.

This determines the complexity of proof search in CKMP:

Corollary 7.8. Provability in CKMP is in PSPACE.

Proof. By Corollary 7.7, it suffices to show that proof search in $\mathrm{CKMP}_{g}^{0}$ is in PSPACE. The latter is shown analogously to Theorem 7.1, as proofs in $\mathrm{CKMP}_{g}^{0}$ are easily seen to have at most polynomial depth.

The same line of reasoning applies essentially without change to CKIDMP, so that provability in CKIDMP is in PSPACE.

The same approach works for logics that include (CEM), with the only actual modification being that in the systems $\mathrm{CKMPCEM}_{g}^{0}$ and $\mathrm{CKIDMPCEM}_{g}^{0}$, backwards application of both $\left(\mathrm{MP}_{g}\right)$ and $\left(\mathrm{MPCEM}_{g}\right)$ is restricted to unmarked formulas. Equivalence of the restricted systems to the full systems is shown in the same manner as for CKMP. We then have, in analogy to Theorem 7.3

Theorem 7.9. Provability in CKMPCEM and in CKIDMPCEM is in PSPACE.

We note that the complexity of CKMPCEM was explicitly left open in [13. We also note that regrettably we were not able to reproduce our claim from [18. that CKMPCEM is in coNP, the problem being that the estimate (7.1) breaks down in the presence of $\left(\mathrm{MP}_{g}\right)$ and $(\mathrm{MPCEM})_{g}$; since no better lower bound than coNP is currently known for CKMPCEM and CKIDMPCEM, this means that the exact complexity of these logics remains open. 


\section{Conclusions}

We have established a generic method of cut elimination in modal sequent system based on absorption of cut and structural rules by sets of modal rules. We have applied this method in particular to various conditional logics, thus obtaining cut-free unlabelled sequent calculi that complement recently introduced labelled calculi [13. In at least one case, the conditional logic CKMPCEM with modus ponens and conditional excluded middle, our calculus seems to be the first cut-free calculus in the literature, as cut elimination for the corresponding calculus in 13 was explicitly left open. We have applied these calculi to obtain complexity bounds on proof search in conditional logics; in particular we have reproved known upper complexity bounds for CK, CKID, CKMP [13] and improved the bound for CKCEM and CKIDCEM from PSPACE to coNP using dynamic programming techniques following [20]. Moreover, we have obtained an upper bound PSPACE for CKMPCEM, for which no bound has previously been published; a strong suspicion remains, however, that this logic is actually in coNP. We conjecture that our general method can also be applied to other base logics, e.g. intuitionistic propositional logic or first-order logic; this is the subject of further investigations.

\section{REFERENCES}

[1] A. Avron and I. Lev. Canonical propositional Gentzen-type systems. In International Joint Conferences on Automated Reasoning, IJCAR 01, vol. 2083 of LNCS, pp. 529-544. Springer, 2001.

[2] J. Burgess. Quick completeness proofs for some logics of conditionals. Notre Dame J. Formal Logic, 22:76-84, 1981.

[3] B. Chellas. Modal Logic. Cambridge University Press, 1980.

[4] A. Ciabattoni, N. Galatos, and K. Terui. From axioms to analytic rules in nonclassical logics. In Logic in Computer Science, LICS 08, pp. 229-240. IEEE Press, 2008.

[5] A. Ciabattoni and K. Terui. Towards a semantic characterization of cut-elimination. Stud. Log., 82:95119, 2006.

[6] C. Cross. Conditional excluded middle. Erkenntnis, 70:173-188, 2009. 10.1007/s10670-008-9146-6.

[7] G. Gentzen. Untersuchungen über das logische Schließen. Math. Z., 39:176-210, 1934.

[8] L. Giordano and C. Schwind. Conditional logic of actions and causation. Artif. Intell., 157:239-279, 2004.

[9] R. Goré. Tableau methods for modal and temporal logics. In M. D'Agostino, D. Gabbay, R. Hähnle, and J. Posegga, eds., Handbook of Tableau Methods, pp. 297-396. Kluwer, 1999.

[10] A. Heuerding, M. Seyfried, and H. Zimmermann. Efficient loop-check for backward proof search in some non-classical propositional logics. In Theorem Proving with Analytic Tableaux and Related Methods, TABLEAUX 1996, vol. 1071 of LNCS, pp. 210-225. Springer, 1996.

[11] S. Kraus, D. J. Lehmann, and M. Magidor. Nonmonotonic reasoning, preferential models and cumulative logics. Artif. Intell., 44:167-207, 1990.

[12] R. Ladner. The computational complexity of provability in systems of modal propositional logic. SIAM J. Comput., 6:467-480, 1977.

[13] N. Olivetti, G. L. Pozzato, and C. Schwind. A sequent calculus and a theorem prover for standard conditional logics. ACM Trans. Comput. Logic, 8(4:22):1-51, 2007.

[14] D. Pattinson and L. Schröder. Cut elimination in coalgebraic logics. Inform. Comput. To appear.

[15] D. Pattinson and L. Schröder. Generic modal cut elimination applied to conditional logics. In Automated Reasoning with Analytic Tableaux and Related Methods, TABLEAUX 09, vol. 5607 of LNCS, pp. 280294. Springer, 2009.

[16] J. Rasga. Sufficient conditions for cut elimination with complexity analysis. Ann. Pure Appl. Logic, 149:81-99, 2007.

[17] L. Schröder and D. Pattinson. Shallow models for non-iterative modal logics. In Advances in Artificial Intelligence, KI 2008, vol. 5243 of LNAI, pp. 324-331. Springer, 2008. 
[18] L. Schröder and D. Pattinson. Pspace bounds for rank-1 modal logics. ACM Trans. Comput. Logic, 10(2:13):1-33, 2009.

[19] L. Schröder, D. Pattinson, and D. Hausmann. Optimal tableaux for conditional logics with cautious monotonicity. In M. Wooldridge, ed., European Conf. on Artificial Intelligence, ECAI 2010, vol. 215 of Frontiers in Artificial Intelligence and Applications, pp. 707-712. IOS Press, 2010.

[20] M. Vardi. On the complexity of epistemic reasoning. In Logic in Computer Science, pp. 243-251. IEEE, 1989. 